\title{
Ancora per la critica del Physiologus Greco.
}

Dall Ambrosiano C, 255 inf. $(=p)$ pubblicai (Studi Italiani di Filologia classica $V$, p. 113-219) quarantotto capitoli di una redazione del Physiologus Greco, la quale potrebbe dirsi Basiliana; e di un' antica versione di essa, dovuta al Pizzimenti, davo, dopo il Teza che vi aveva accennato stampandone un capitolo, un saggio in una pubblicazione per nozze $D e$ Fernex $=$ Wuille-Bille. Presentando però la versione del Pizzimenti in confronto di $p$ un numero minore di capitoli e potendo sorgere il dubbio che ciò si debba non ad ommissione del traduttore ma al ms. su cui venne condotta la versione stessa, ed essendo d' altra parte $p$ il solo ms. noto come contenente la redazione Basiliana, era naturale che nei mss. del Physiologus non ancora investigati si andassero cercando somiglianze $o$ identità rispetto a $p$, e non sarà discaro, ritengo, il sapere che abbiamo un ms. identico a $p$ nel codice greco 68 a della Biblioteca Capitolare di Viterbo. ${ }^{1}$ )

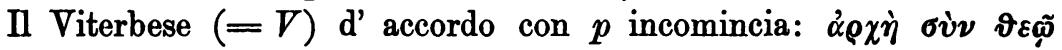

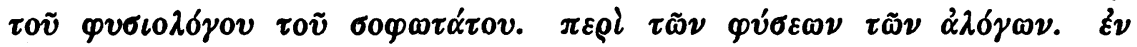

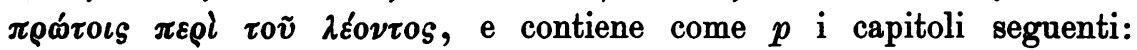

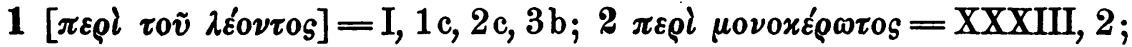

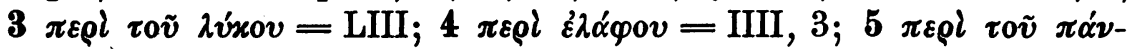

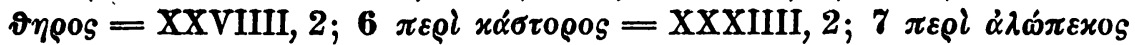

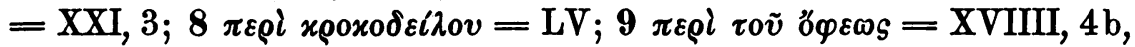

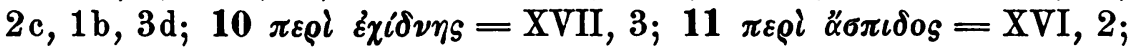

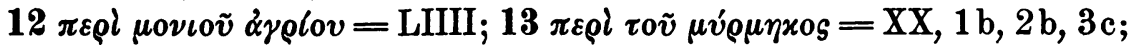
$14 \pi \varepsilon \rho l \beta \alpha \tau \rho \alpha ́ \chi \omega \nu=X X X X I I I, 3 ; 15 \pi \varepsilon \rho i ~ \alpha \varepsilon \tau 0 \tilde{v}=\mathrm{VI}, 1,2 \mathrm{a}, 3 ; 16 \pi \varepsilon \rho i$

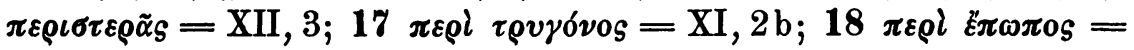

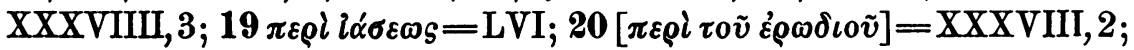

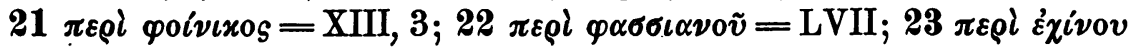
$=$ XXVIII, 3; $24 \pi \varepsilon \rho i$ rov $\lambda \alpha \gamma o \tilde{v}=$ LVIII; $25 \pi \varepsilon \rho i \quad \chi \alpha \lambda \alpha \nu \delta \rho i o v=$

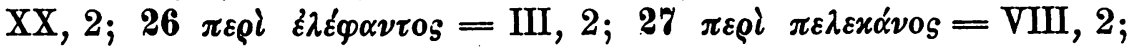

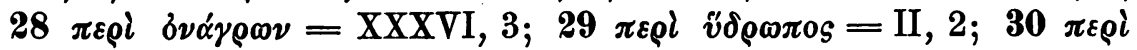

1) Vedine la descrizione in L. Dorez, Latino Latini et la biblioth. cap. de Viterbe, in Rev. des Biblioth. 1895 p. 251. 


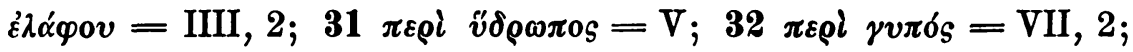

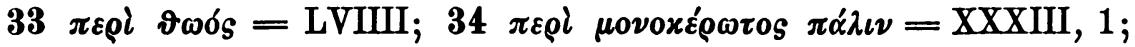
$35 \pi \varepsilon \rho i$ iं

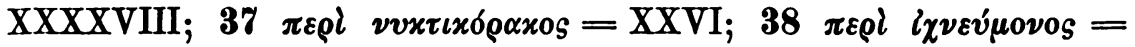

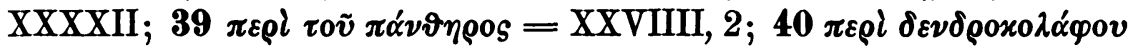

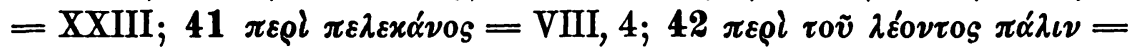

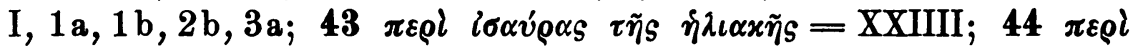

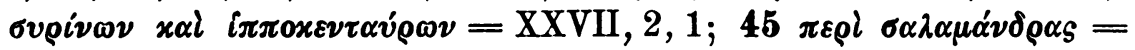

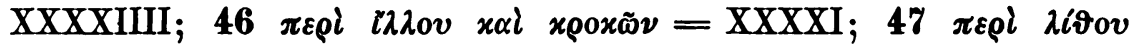

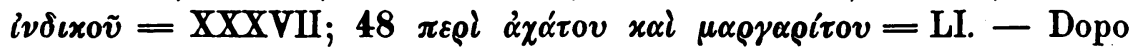

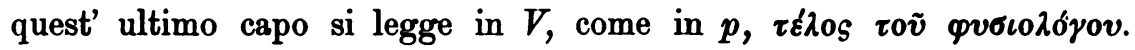
$\tau \tilde{\boldsymbol{\varphi}} \boldsymbol{\vartheta} \varepsilon \tilde{\boldsymbol{j}} \delta \delta \hat{\xi} \alpha$.

Le differenze, per usare questo vocabolo, fra $V$ e $p$ sono essenzialmente grafiche.

$\alpha)$ In $p$ è molto più raro che in $V$ lo iota sottoscritto, mentre è più frequente l' uso dei consueti compendii per i varii casi di aै $\nu \vartheta \rho \omega \pi 0 s$,

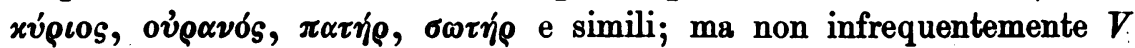
e $p$ per queste parole hanno nei medesimi luoghi concordemente il compendio, in altri luoghi concordemente scrivono il vocabolo per disteso. In $p$ abbiamo qualche esempio di abbreviazioni e di compendii in fine di parola, che invece in $V$ sono molto rari, sebbene generalmente $V$ e $p$ coincidano anche per questo rispetto: $2=\mathrm{XXXIII,} \mathrm{2,} \mathrm{1.} 5$ i $\pi \alpha \alpha \varrho \mathrm{e}$

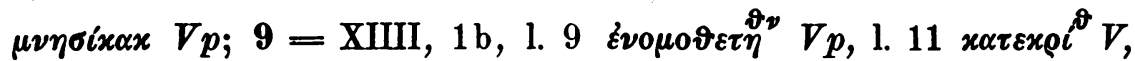
${ }_{x / \alpha \tau \varepsilon \tau \rho \iota}^{\wedge} p$ e $\eta^{\tau} \cdot V p ; 13=\mathrm{XX}, 1 \mathrm{~b}, 1.18 \beta 0 v \lambda_{\eta}^{\vartheta} V p ; 15=\mathrm{VI}, 3,1.8$

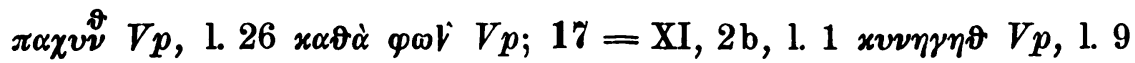

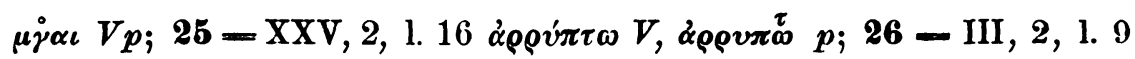
$\delta \iota \alpha \gamma \omega \vartheta \eta V, \delta \iota \alpha \gamma \omega \vartheta \eta^{\vee} p$, l. 63 ö $\tau \iota \dot{\eta} \dot{\alpha} \mu \alpha \rho \tau V p ; 27=$ VIII, 2, 1. 6 $\beta \alpha \sigma \iota \lambda V p ; 30=$ IIII, 2, l. $1 \alpha v \dot{v}$. Nella mia trascrizione di $V$ e di $p$ non ho notato tutti i luoghi ne' quali riguardo a questo fatto ci sia una qualche differenza; mi limito a citare i seguenti passi, che possono

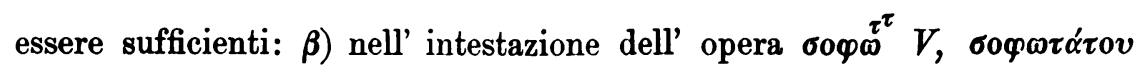

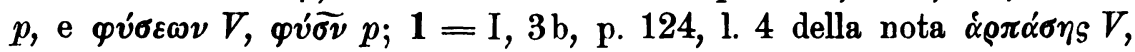
$\dot{\alpha} \rho \pi \alpha \sigma^{\varsigma} p ; 4=\mathrm{IIII}, 3$, l. $10 \ddot{\alpha} \nu \varepsilon \sigma \iota \nu V, \alpha^{\prime} \nu \varepsilon \sigma^{\wedge} p ; 14=\mathrm{XXXXIII,} \mathrm{3,} 1.15$

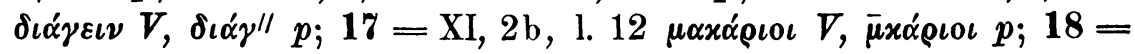

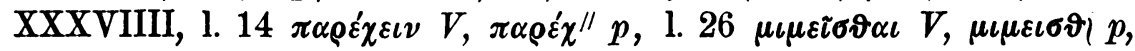

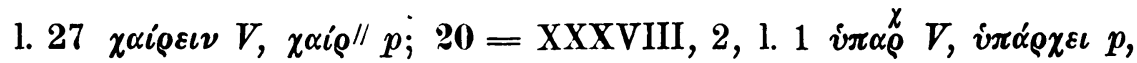
1. $16 \alpha \dot{v} \tau \tilde{\omega} \nu V, \alpha \dot{v} \tilde{\tau} p ; 21=\mathrm{XIII}, 3,1.4 \tau \dot{\eta} \nu V, \hat{\tau} p, 1.11 \pi \rho 0$ t $V$, 


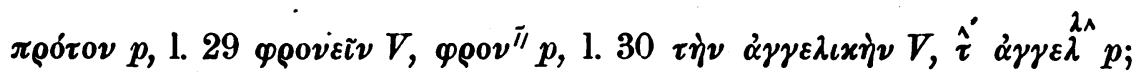
$22=\mathrm{LVII} \dot{\alpha} \pi 0 \nu \varepsilon 0 \sigma \sigma \omega \dot{\sigma \varepsilon \iota \nu} \nabla, \dot{\alpha} \pi 0 \nu \varepsilon 0 \sigma \sigma \sigma^{\top} p ; 25=\mathrm{XXV}, 2,1.8$

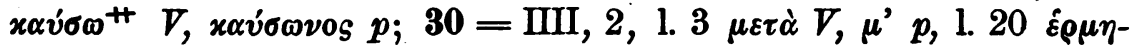

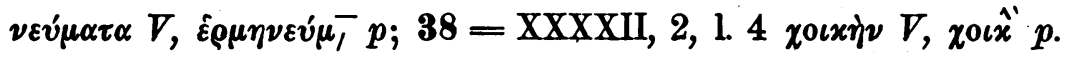

Nè costituiscono differenza gli errori simili ai seguenti: $\gamma$ ) $1=\mathrm{I}$,

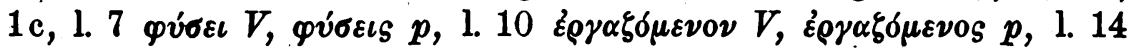

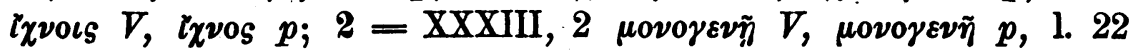

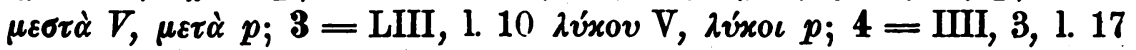

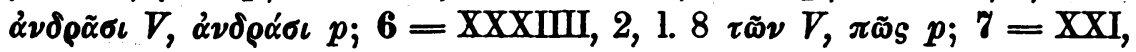

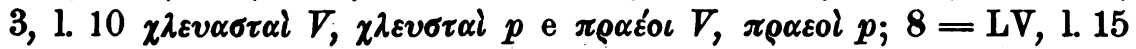

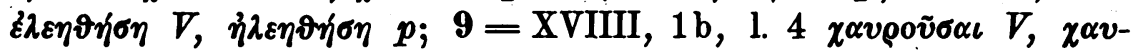

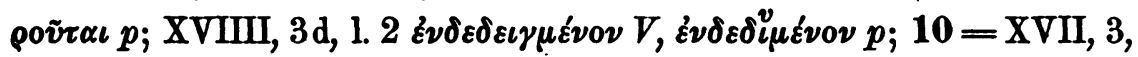

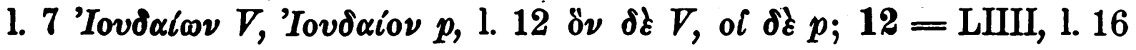

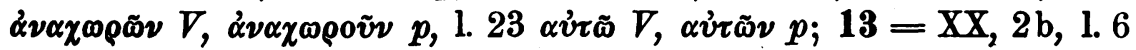

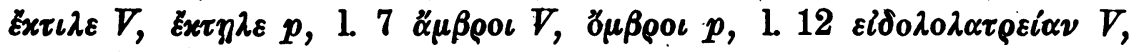

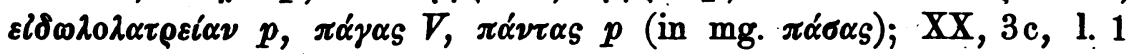

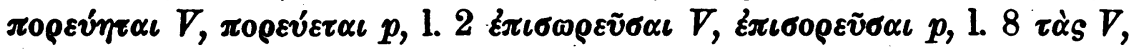

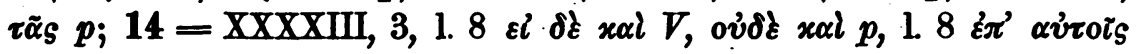

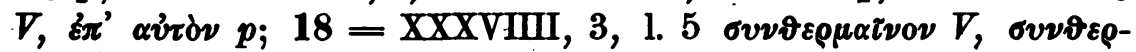

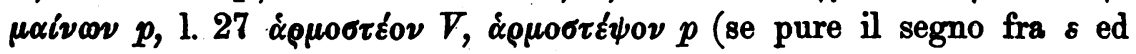
o non si dere intendeve cancellato dalla linea verticale della lettera che

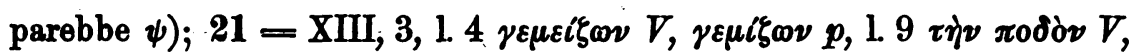

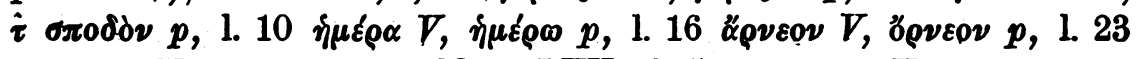

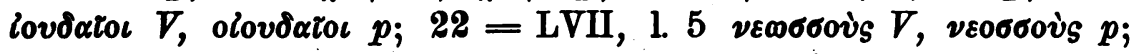

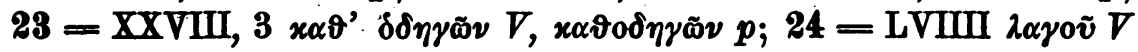
nell' intestazione, $\lambda \alpha \gamma \omega \circ \tilde{v} p$ in margine; $26=\mathrm{III}, 2,1.17 . \alpha \pi 0 \pi \lambda \varepsilon^{\xi} \xi \alpha-$

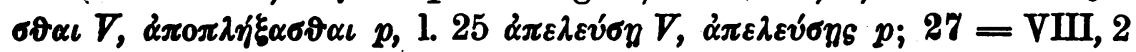

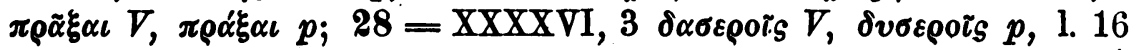

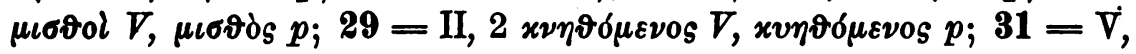

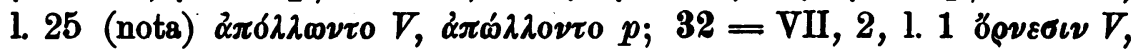

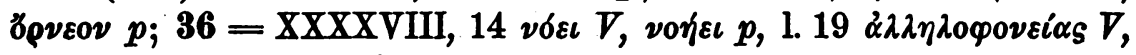

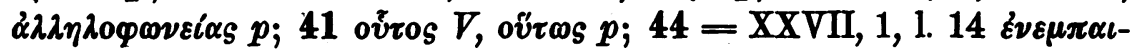

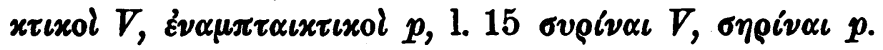

$\mathrm{Ma}$ il fatto piu importante è questo che $p$ presenta frequenti correzioni interlineari e marginali, ed in questi casi $V$ dà generalmente

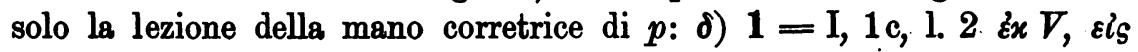

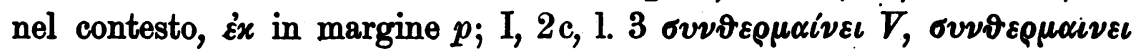

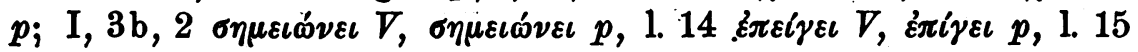

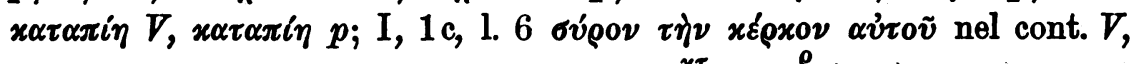

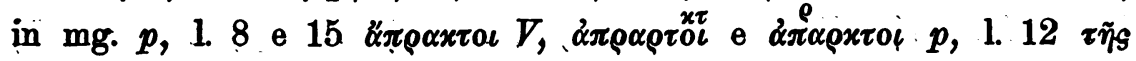




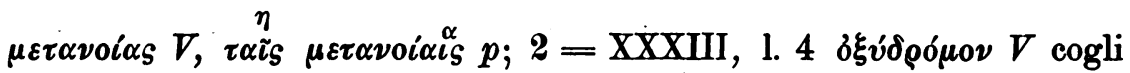
accenti cancellati da lineetta trasversale, $\partial \xi v \delta \rho \sigma \mu \varepsilon \nu 0 \nu$ nel cont., $\delta \rho \circ \mu \nu \nu$

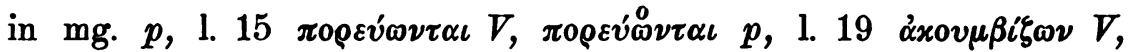

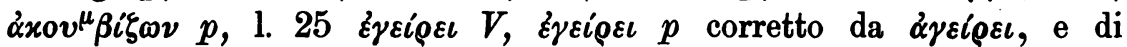

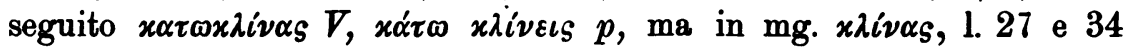
$\tau \rho \varepsilon \pi \tau \iota x \tilde{\omega} s \quad V$,

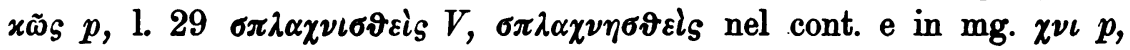

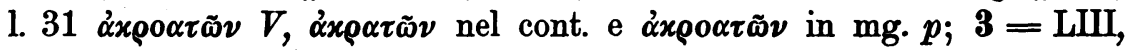

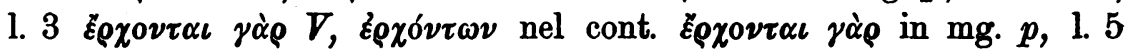

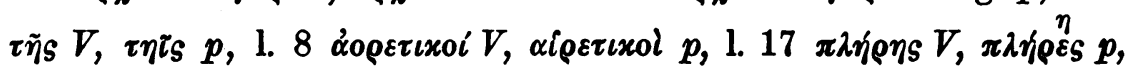

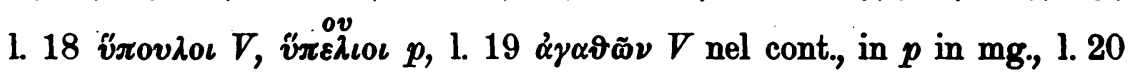

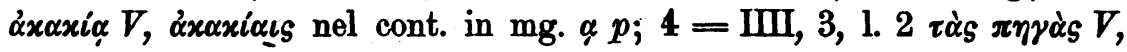

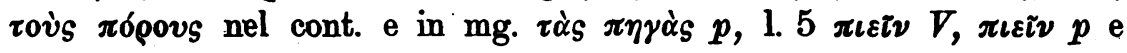

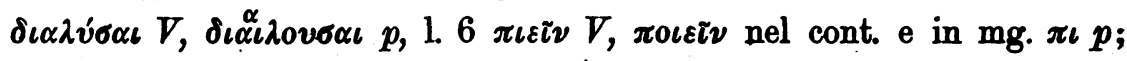

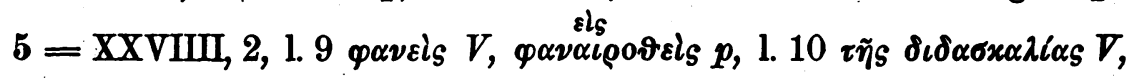

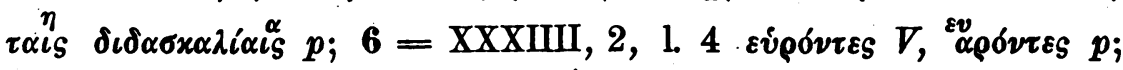

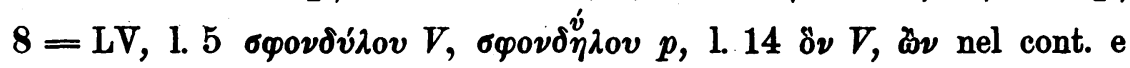

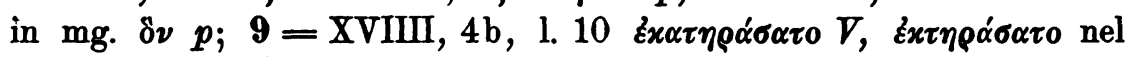

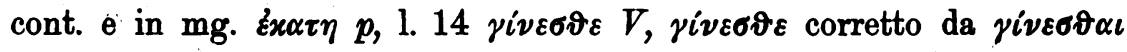

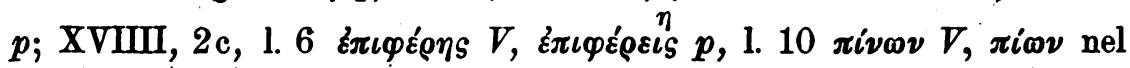

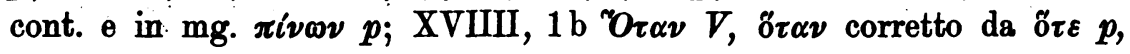

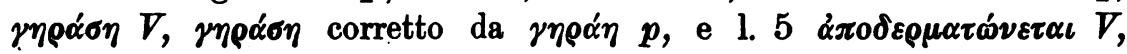

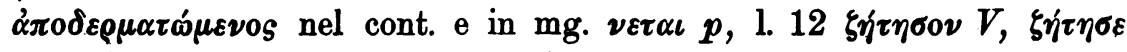

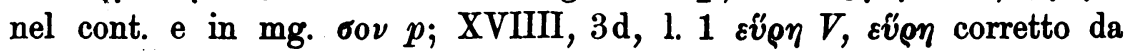

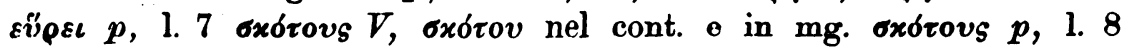
$\beta \iota \omega \tau \iota x \tilde{\nu} \nu V, \beta \iota \dot{\tau} \tau 0 \nu$ nel cont. $\mathrm{e}$ in mg. $\tau \iota x \tilde{\omega} \nu p ; 10=\mathrm{VII}, 3,1.4$

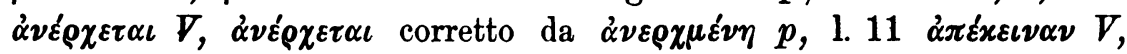

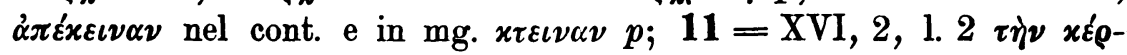

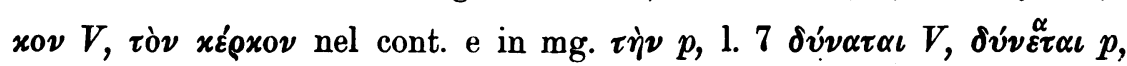

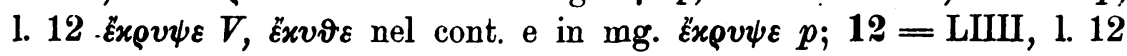

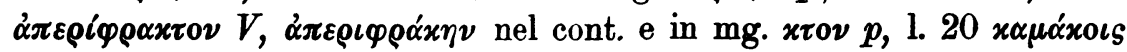

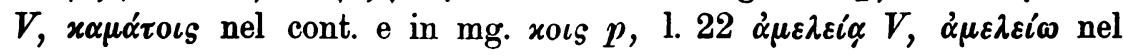
cont. e in mg. $\lambda \varepsilon i \alpha, p ; 13=\mathrm{XX}, 1 \mathrm{~b}, 1.1 \mu i \mu \eta \sigma \alpha \iota V, \mu i \mu \eta \sigma \varepsilon \varepsilon^{\alpha \iota} p, 1.10$

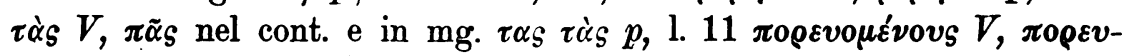

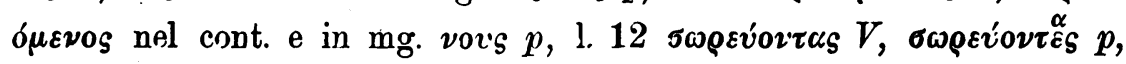

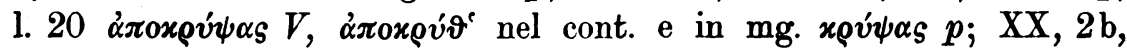

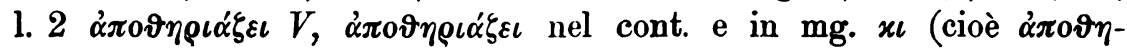




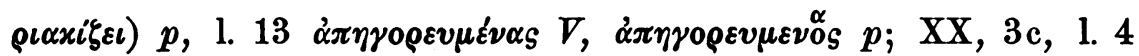

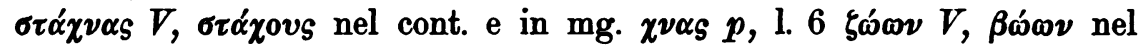

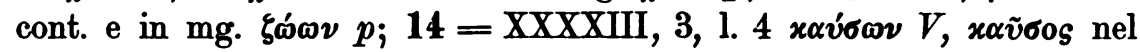

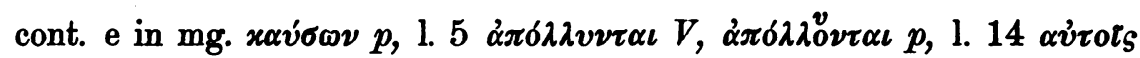

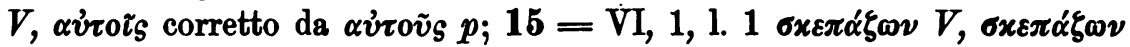

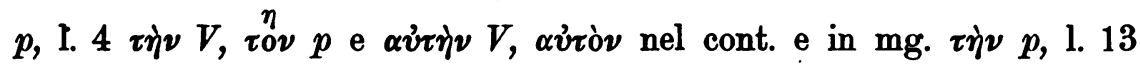

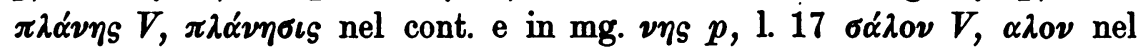

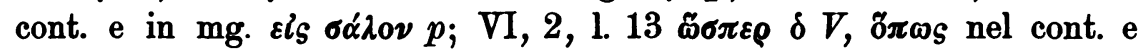

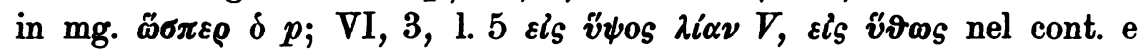

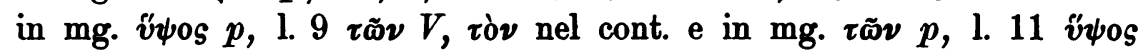

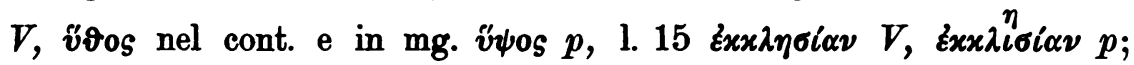

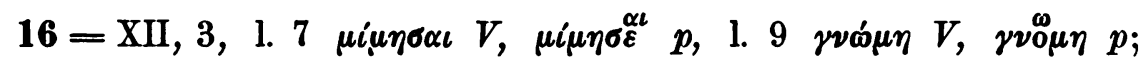
$17=\mathrm{XI}, 2 \mathrm{~b}, 1.5 \mu 0 \nu \alpha \nu \delta \rho i \alpha \nu V, \mu 0 \nu \alpha \nu \delta i \alpha \nu$ nel cont. e in mg. $\delta \rho i \alpha \nu p$,

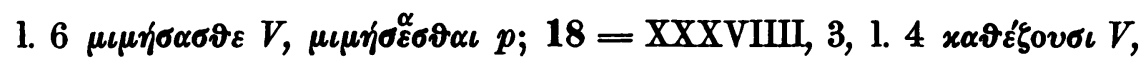

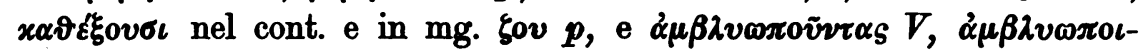

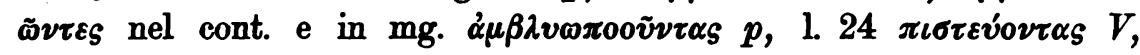

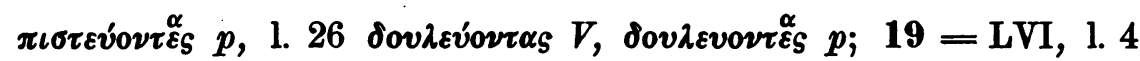

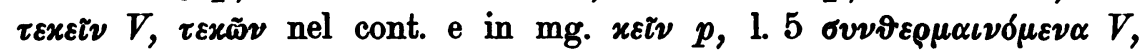

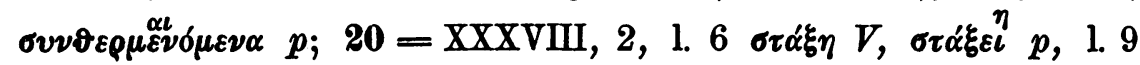

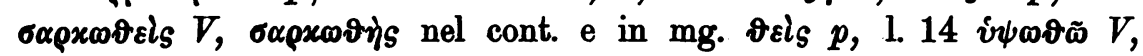

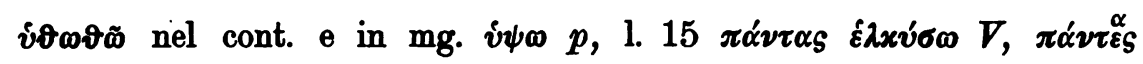

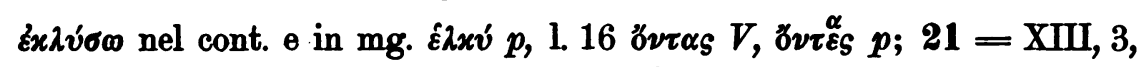

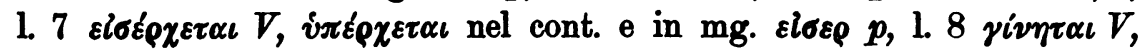

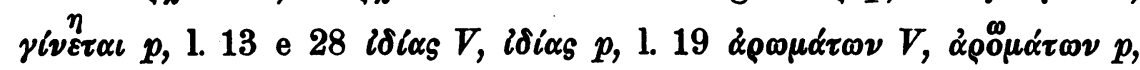

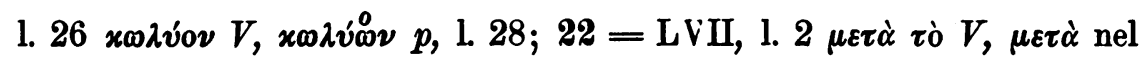

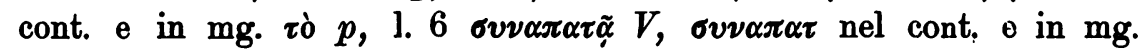

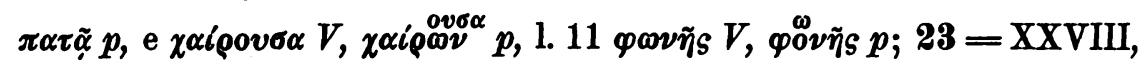

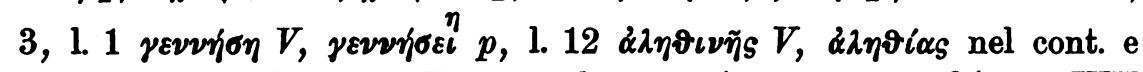

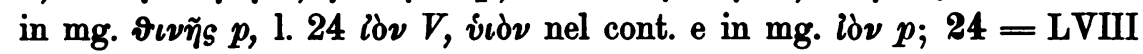

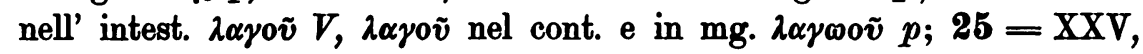

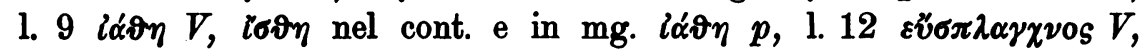
$\varepsilon \dot{v} \sigma \pi \lambda \alpha \gamma \chi \nu \delta \mu \varepsilon \nu 0 s$ nel cont. $\theta$ in mg. $\varepsilon \ddot{v} \sigma \pi \lambda \alpha \gamma \gamma \circ \mathrm{s} p, 1.13 \dot{\alpha} \pi 0 \sigma \tau \rho \alpha \varphi \eta_{s} V$,

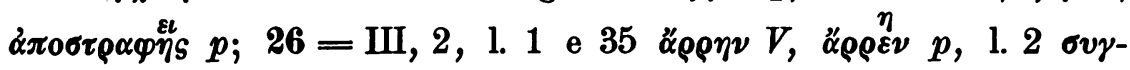

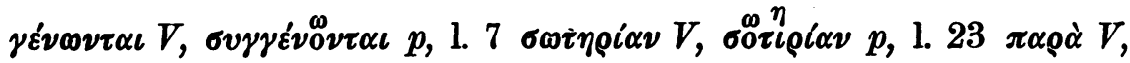
$\pi \alpha \rho \dot{\alpha}$ $\boldsymbol{d} \dot{\varepsilon}$

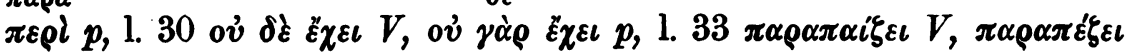




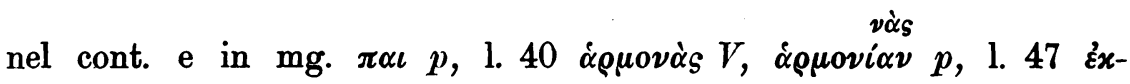

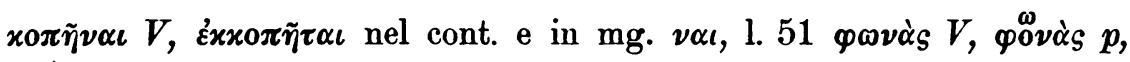

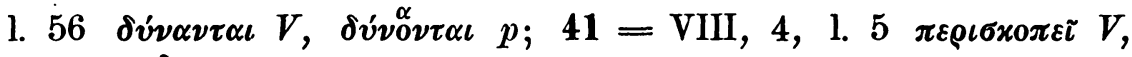
$\varepsilon \bar{\imath}$ $\pi \varepsilon \rho \iota \sigma x о \pi \omega \nu$ p.

Non costituiscono certamente differenza altri fenomeni come i seguenti: $\varepsilon$ ) $3=\mathrm{LIII}, 1.8^{~} E_{\rho} \mu \eta \nu \varepsilon i \alpha$ omm. $V ; 11=\mathrm{XVI}, 2,1.6 \dot{\omega}_{S}$

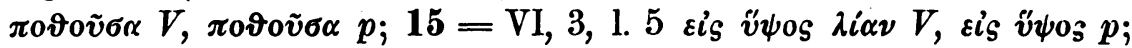

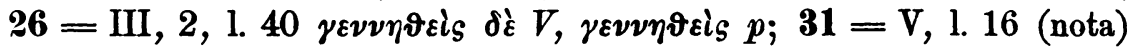

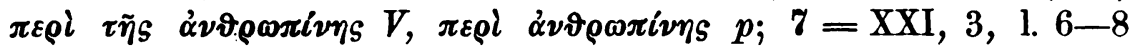

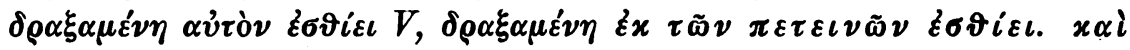

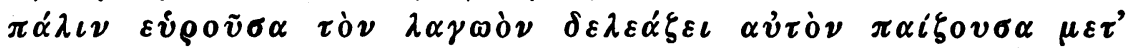

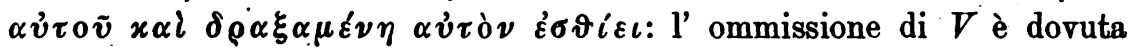
ad omoioteleuto, mentre quelle di $p$ or ora vedute ai capitoli 11, 15, 26, 31 paiono causate dalla presenza de' soliti compendii, che l'amanuense di $p$ non seppe risolvere, ovvero da errori ora di $V$ ora di $p$, ovvero anche da non retta lettura dell' archetipo; ed eccone una

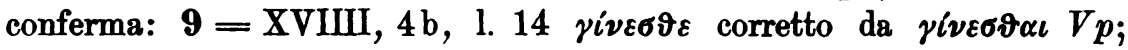

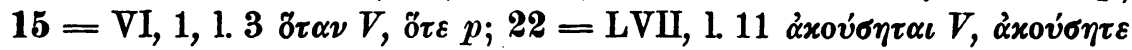

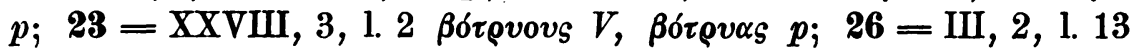

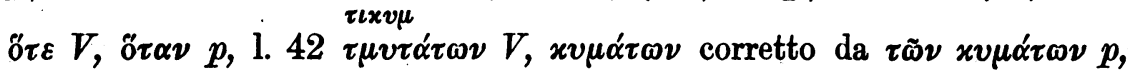

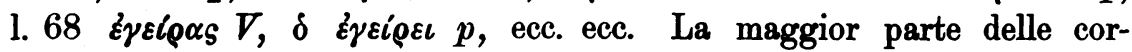
rezioni interlineari e marginali di $p$ hanno questa origine.

A stabilire la massima somiglianza fra $V$ e $p$ possono bastare $\mathrm{i}$ dati finora veduti; da essi si può dedurre che $p$ non è copia di $V$, nè $V$ di $p$, ma che derivano entrambi dal medesimo archetipo. Che $p$ non sia copia di $V$ si dimostra facilmente: $V$ è scritto chiarissimo, sicchè la lettura ne è facile e sicura e tale da eliminare qualsiasi possibilità agli errori di $p$ corretti fra le linee e sul margine, errori e correzioni di siffatta natura da escludere anche il dubbio che l' amanuense di $p$ trascrivesse $V$ non leggendolo ma scrivendo sotto dettatura; cfr. p. es. $13=\mathrm{XX}, 2 \mathrm{~b}, \mathrm{l} .2$ ove la correzione marginale fa supporre che il ms. onde si trascrisse $p$ desse $o$ inducesse a leggere $\dot{\alpha} \pi 0 \vartheta \eta \rho \iota \alpha x l \xi \varepsilon \iota$ o $\dot{\alpha} \pi 0 \vartheta \eta \rho i \xi \varepsilon \iota$. Nè $V$ è copia di $p$, come, anche astraendo da ragioni cronologiche, è ampiamente dimostrato dei fatti raccolti sotto le categorie $\alpha), \beta), \varepsilon)$. Nè $p$ fu riveduto su $V$, chè alcuni errori e alcune buone lezioni di $V$ sarebbero così entrate in $p$ : di più, le divergenze, in bene e in male, fra $V$ e $p$ corrispondono spesso alle lezioni di altri mss., che non dando l' Hermeneia Basiliana espongono le varie nature in modo simile o prossimo a quello della redazione Basiliana. Rimane 
dunque una sola ipotesi, che $V$ e $p$ derivino da fonte comune, come

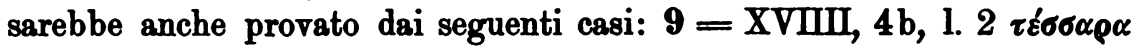

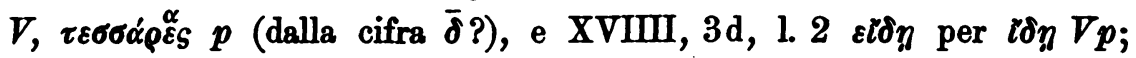

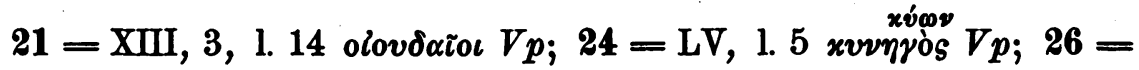

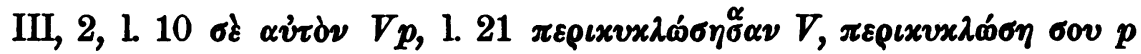
( $V$ e $p$ trascrivono un luogo che presentava difficoltà e poca chiarezza $\tau \iota x v \mu$

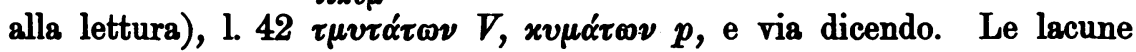
di $V$ e $p$ sono le stesse, hanno eguale estensione ed eguale disposizione materiale nelle righe: un luogo istruttivo è $41=$ VIII, $4,1.3$, dove $\alpha l_{p \varepsilon \varepsilon}$

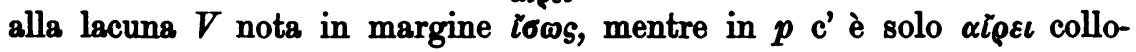
cato dopo vँ\&os. Sembra dunque che si tratti di trascrizione da ms. che non presentasse lacune, ma che in molti punti fosse difficile alla lettura, sicchè talora nulla più si riuscisse a decifrare, tal' altra le lettere molto evanide si prestassero alla trascrizione secondo la diversa abilità dell' amanuense; cfr. $47=\mathrm{XXXVII}, 1.4$ (nota) $\tau \dot{\partial} \nu \lambda i$ ivov $x \alpha i$

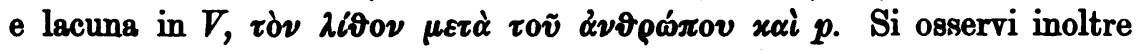
che in $V$ e $p$ il testo comprende presso che il medesimo numero di pagine del medesimo formato, che spesso l' intestazione e la disposizione delle righe è la medesima, tanto che conoscendo $V$ si conosce $p$ e vice versa, e l' un ms. pud far le veci dell' altro, pur avendo $p$ il vantaggio di indicare la divisione fra la parte ove compare quasi costantemente il nome di Basilio, da quella ove esso nome è sporadico.

Le relazioni fra $V$ e $p$ possono maggiormente essere comprese studiandone le relazioni colla versione del Pizzimenti, che designo sempre con $P$, e col testo greco che essa ebbe a fondamento (che indico con $x$ ), quale dalla versione si pud desumere che fosse.

II

Ecco l' indice di $P$ confrontato con quello di $p: 1$ del leone (=1); 2 del monocerote $(=2) ; 3$ del lupo $(=3) ; 4$ della cerva $(=4) ; 5$ della panthera $(=5) ; 6$ del castore $(=6) ; 7$ della volpe $(=7) ; 8$ del crocodilo $(=8) ; 9$ del serpente $(=9) ; 10$ della vipera $(=10) ; 11$ dell' aspido (=11); del cinghiale (=12); 13 della formica $(=13) ; 14$ delle rane (=14); 15 dell' aquila (=15 ma solo per VI, 1 e VI, 2a); 16 della colomba $(=16) ; 17$ della tortora $(=17) ; 18$ dell upopa (=18); 19 del giasi $(=19) ; 20$ dell' erodio $(=20) ; 21$ della phenice (=21); 22. del fagiano (=22); 23 del riccio (=23); 24 della lepre (=24); 25 del chalandrio (=25); 26 dell' elephante $(=26) ; 27 \cdot$ del pelicano (=27 e 41); 28 dell' asino selvaggio (= 28); 29 dell' hydrope 
(= 29); 30 della cerva $(=30) ; 31$ dell' hydrope (= 31); 32 dell' aquila (= 15 solo per VI, 3); 33 dell' avoltoio (= 32); 34 del monocerote $(=34) ; 35$ delle pietre di foco $(=35) ; 36$ del prione $(=36)$; 37 del notticorace $(=37) ; 38$ dell ichneumone $(=38) ; 39$ della panthera (= 39); 40 del picchio (= 40).

Oltre alle differenze palesi dal titolo dei capitoli parecchie ne troviamo fra $P$ e $V p$; taluna è dovuta al desiderio di conseguire nella traduzione chiarezza ed eleganze ed eziandio esattezza: $1=\mathrm{I}, 1 \mathrm{c}, 1.2$

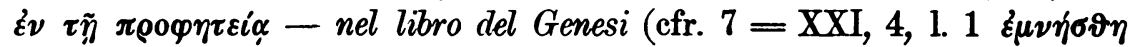
$\Delta \alpha v i \delta$ ċv roĩs $\psi \alpha \lambda \mu o i_{s}$ - fè mentione Christo nelli evangelij; $16=$

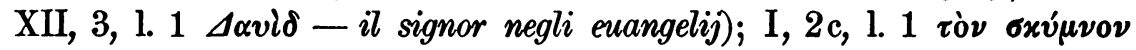

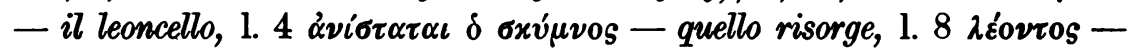

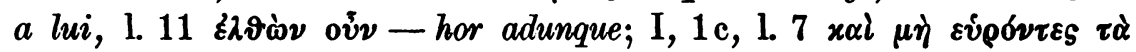

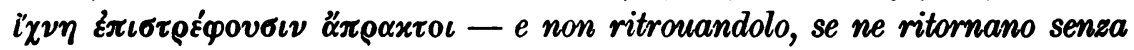

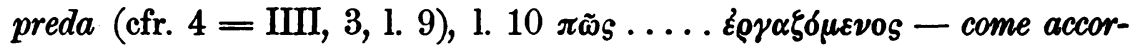

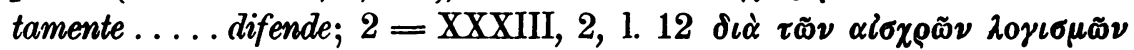

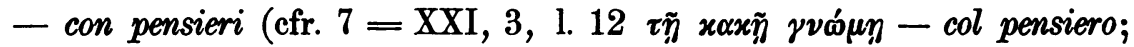

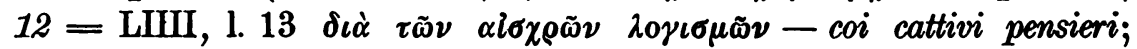

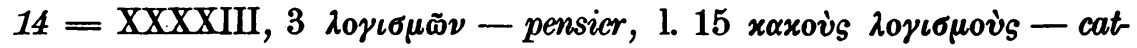

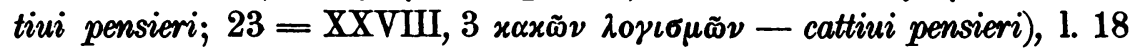

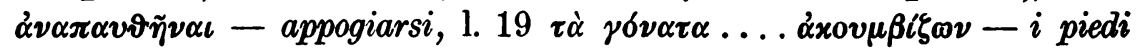

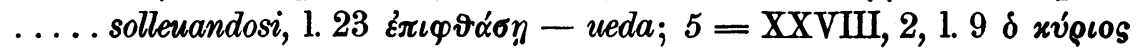

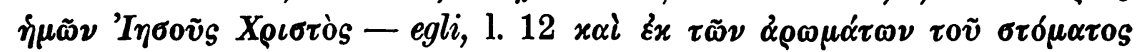

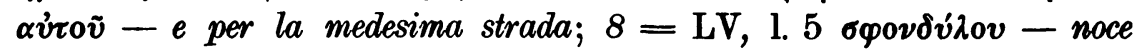

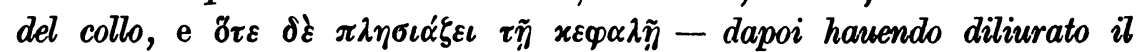

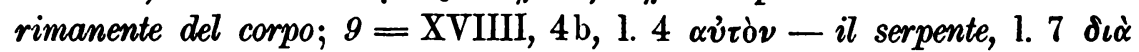

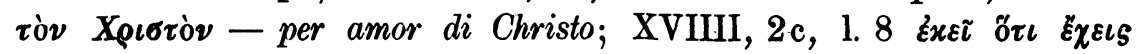
$\tau \iota v \alpha$ x $x \alpha \tau \alpha$ ' $\sigma 0 v$ - che il tuo fratello ha qualche odio contro di te, 1.10

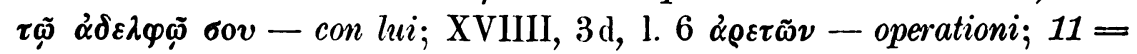

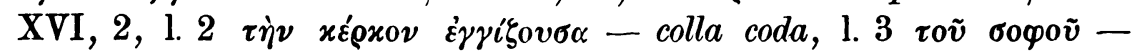
dell' incantatore (nel passo biblico citato, come quasi sempre, in latino, troviamo: uenefici incantantis sapienter; cfr. 1.7 бo甲òs - incantatore),

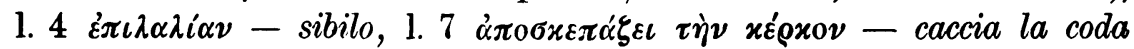

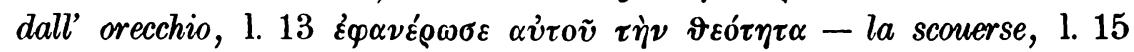

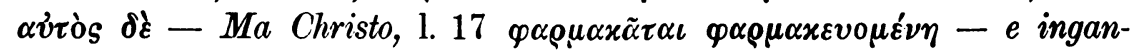

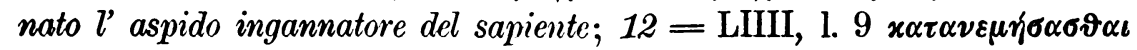

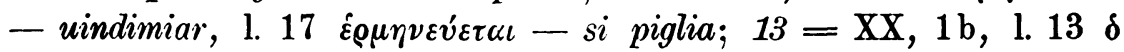

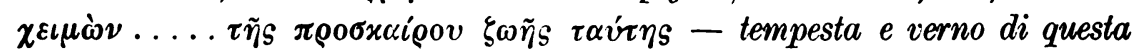

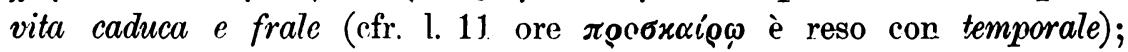
$\mathrm{XX}, 2 \mathrm{~b}, 1.2 x \alpha \tau \alpha \lambda \dot{\alpha} \beta \omega \sigma \iota$ - cogliono la formica, $\varepsilon^{2} x \beta \alpha \dot{\alpha} \lambda \lambda \varepsilon \iota$ - caccia fuori

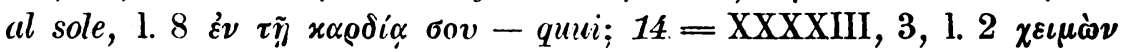
Byzant Zeitschrift IX 1. 


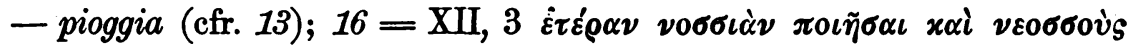

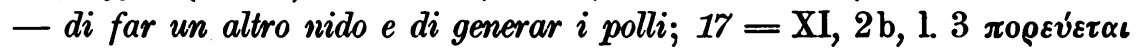

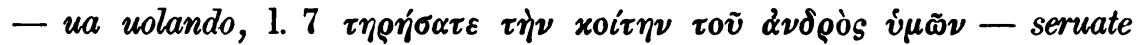
castità ài uostri mariti, $1.10 \delta$ จ

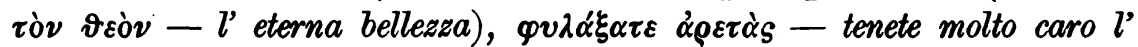

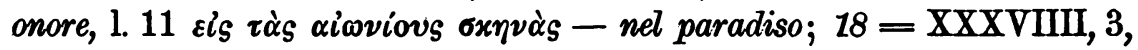

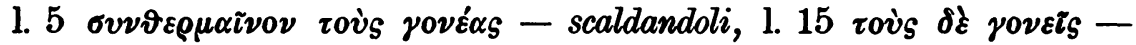

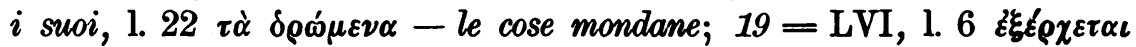

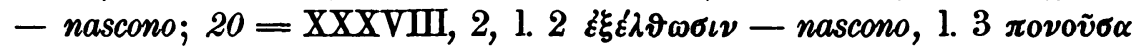

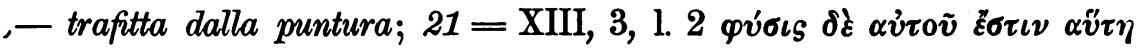

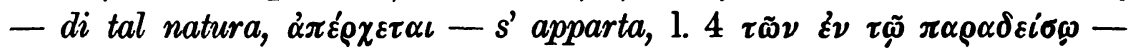

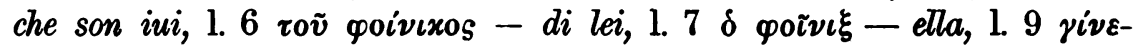

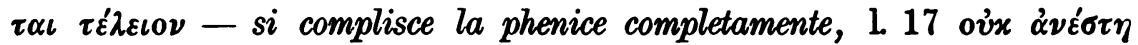

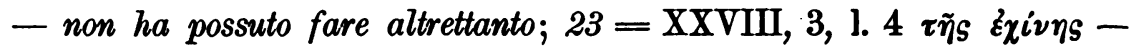

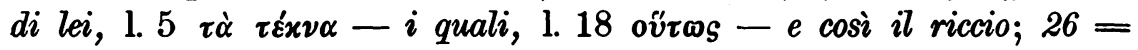

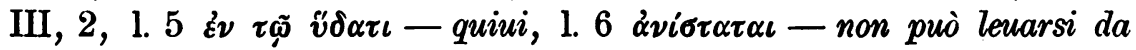

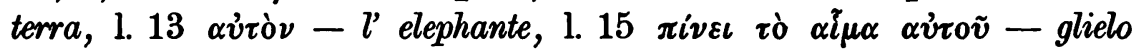

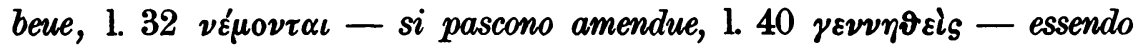
partorito il figlio, 1. $44 \pi \alpha \varrho \alpha ́ x \lambda \varepsilon \iota \tau \alpha-g r a n d i$ (cfr. 1. 63), 1. 47 Exxxo-

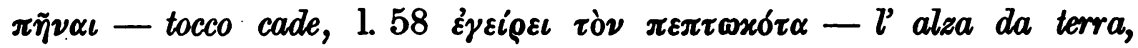

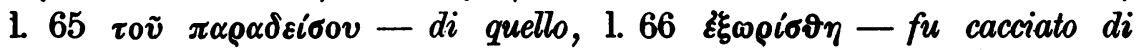

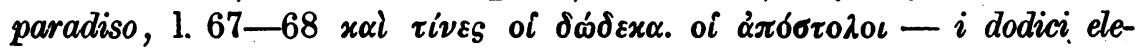

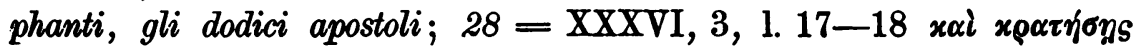

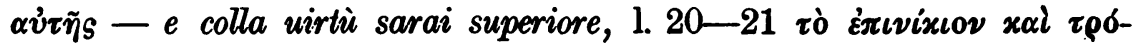
$\pi \alpha \iota \mathrm{v}-l e$ ricche spoglie e gli honorati trophei; $29=\mathrm{II}, 2,1.12 \varepsilon i_{S}$

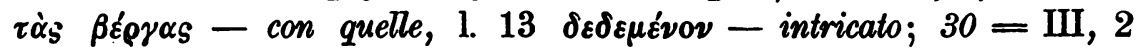
$.0 \nu 0 \mu \alpha^{\prime} \xi \tau \alpha \iota$ - si chiama in greco, $1.19 \pi \eta \gamma \dot{\alpha} \mathrm{s}=$ diuine fontane; $31=$

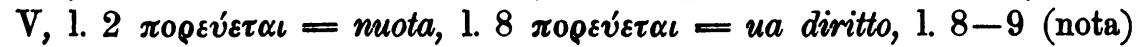

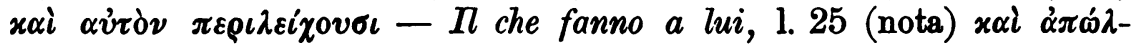

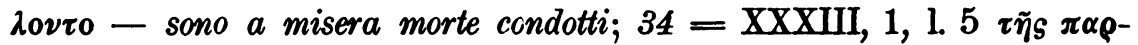

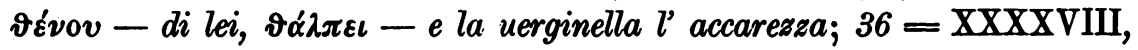
1. $1 \pi \tau \dot{\varepsilon} \rho v \gamma \alpha_{S}$ - penne (cfr. il nostro pinne), ma l. $2 \pi \tau \varepsilon \dot{\rho} v \gamma \alpha_{S}-a l i$,

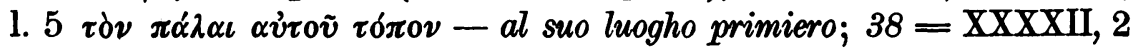

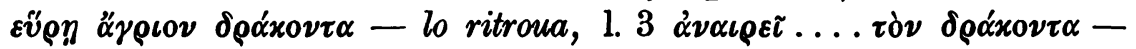

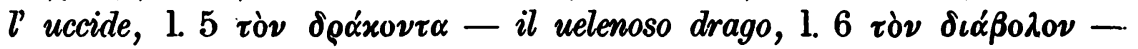
$l$ 'infernal tiranno; $40=\mathrm{XXIII}, 1.2 \pi 0 \lambda v \pi 0$ xilos - d' assai diverse

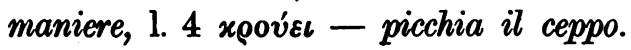

Affini a queste vi sono alcune differenze costanti o quasi, ad es.

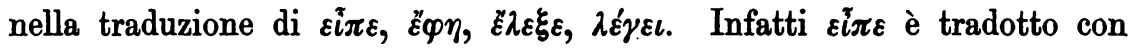
dice in $1=\mathrm{I}, 1,3 \mathrm{~b}, 1.1 ; 2=\mathrm{XXXII}, 2,1.27 ; 3=\mathrm{LIII}, 1.8$ e 18 ; $11=\mathrm{XVI}, 2, \mathrm{l} .10 ; 15=\mathrm{VI}, 1, \mathrm{l} .9 ; 16=\mathrm{XII}, \mathrm{l} .6 ; 17=\mathrm{XI}, 2 \mathrm{~b}, 1.6$; 
$22=$ LVII, $1.7 ; 23=$ XXVIII, 3 , 1.7 e $20 ; 36=$ XXXXVIII, I. 1 ; e con disse in $5=\mathrm{XXVIII}, 2, \mathrm{l} .7 ; 6=\mathrm{XXXXIIII,} \mathrm{l.} 6 ; 7=\mathrm{XXI}, 3$, l. $9 ; 12=\mathrm{LIII}, 1.9 ; 13=\mathrm{XX}, 1 \mathrm{~b}, 1.3 ; 2 \mathrm{~b}, 1.6 ; 3 \mathrm{c}, 1.7 ; 14=$ XXXXIII, $2,1.6 ; 16=\mathrm{XII}, 3,1.7 ; 18=\mathrm{XXXVIIII,} \mathrm{3,} \mathrm{1.} \mathrm{9;} 20=$ XXXVIII, $2,1.8 ; 24=\mathrm{LVIII}, 1.6 ; 25=\mathrm{XXV}, 2,1.10 ; 26=\mathrm{III}, 2$, l. 7 e $20 ; 27=$ VIII, 2 , l. 6 ; $28=\mathrm{XXXVI}, 3,1.6 ; 33=$ VII, 2, 1. 9; e con ha detto in $10=\mathrm{XVII}, 3,1.1$. Ed $\tilde{\varepsilon} \varphi \eta$ è reso con disse in $2=\mathrm{XXXIII}, 2$, l. 32; e con parla in $9=\mathrm{XVIUI}, 4$ b, l. 2. Ed $\varepsilon \lambda_{\varepsilon} \xi \varepsilon$ è tradotto con disse in $6=\mathrm{XXXIII}, 2,1.1 ; 8=\mathrm{LV}, 1.1 ; 12=$ LIIII, 1. 12; $18=\mathrm{XXXVWI,} \mathrm{3,} \mathrm{1.} \mathrm{1;} 19=\mathrm{LVI}, 1.1 ; 21=\mathrm{XIII}, 3$, l. $1 ; 22=\mathrm{LVII}, \mathrm{l} .1 ; 23=\mathrm{XXVIII}, 3,1.15 ; 25=\mathrm{XXV}, 2,1.1$; e con dice in $3=\mathrm{LIII}, 1.4$; e con scrive in $4=\mathrm{III}, 3,1.3$ ed 11; $5=\mathrm{XXVII}, 2,1.1$. E $\lambda \varepsilon^{\prime} \gamma \varepsilon \iota$ con disse in $7=\mathrm{XXI}, 3,1.2$; e con scrisse in $35=\mathrm{XXXXIII,} \mathrm{2,} \mathrm{l.} 1$; e con dice in $9=\mathrm{XVIII}, 4 \mathrm{~b}, 1.6$; $1 \mathrm{~b}, 1.6 ; 3 \mathrm{~d}, 1.5 ; 10=\mathrm{XVII}, 3, \mathrm{l} .3$ e $6 ; 21=\mathrm{XIII}, 3,1.14 ; 22=$ LVII, l. 10;39=XXVIIII, 1, l. 1. Ciò si collega a qualche incertezza, d' altronde spiegabilissima, nell' uso dei tempi; cfr. $13=\mathrm{XX}, 3 \mathrm{c}$, 1. $14 \xi \eta \tau \eta \dot{\sigma} \sigma \nu \tau \varepsilon S$ - cercano, l. $15 \xi \tilde{\omega} \sigma \iota \nu-u i s s e r o ; 15=\mathrm{VI}, 1,1.1$

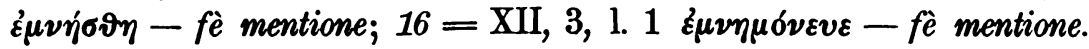

In altri luoghi la differenza è voluta espressamente e sistematicamente, p. es. a principio dei capitoli, ove viene introdotta una certa uniformità, combinata talvolta a desiderio di chiarezza: $1=\mathrm{I}, 3 \mathrm{~b}, 1.1$

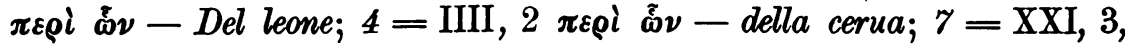

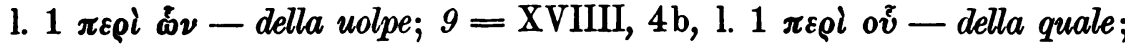

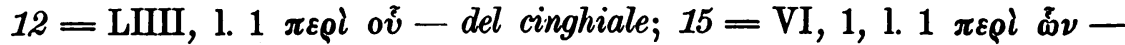
dell' aquila; $16=\mathrm{XII}, 3,1.1 \pi \varepsilon \rho i \tilde{\omega} \nu$ - della colomba; $19=\mathrm{LVI}, 1.1$

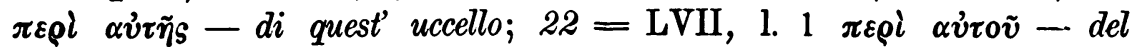

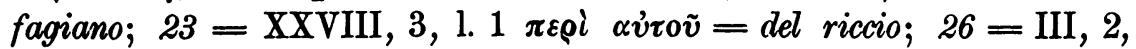

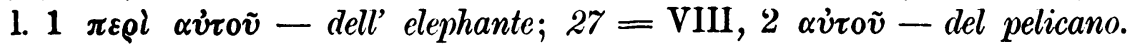
Così pure le intestazioni delle nature hanno forme come le seguenti: ha seconda natura del medesimo in $2=\mathrm{XXXIII,} \mathrm{2,} 1.14 ; 3=\mathrm{LIII}$, 1. $15 ; 9=\mathrm{XVIII}, 2 \mathrm{c}, \mathrm{l} .1 ; 23=\mathrm{XXVIII}, 3,1.17 ; 26=\mathrm{III}, 2,1.13$; $2 \gamma=$ VIII, 4 ; ha seconda natura della medesima in $4=$ IIII, 3, l. 11; $13=\mathrm{XX}, 2 \mathrm{~b} ; 15=\mathrm{VI}, 2,1.1$; ha terza natura del medesimo in $26=$ III, 2, 1. 27.

Altre volte si tratta di traduzione libera: $1=\mathrm{I}, 1 \mathrm{c}, 1.4$ l' $\chi$ vos

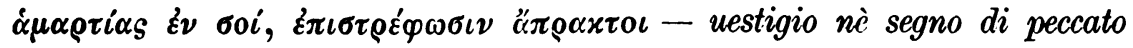
in te, senza seguire al pensiero lo scellerato effetto, se ne uadano uia;

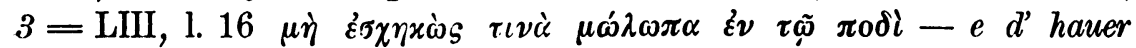

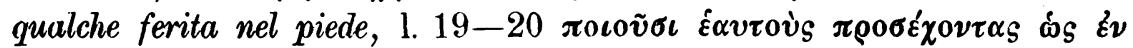

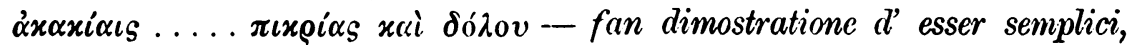


et innocenti .....d inganno e di magagna; $4=\Pi_{\mathrm{I}} \mathrm{I}, 3,1.18$ घ่v $\dot{\alpha} \rho \varepsilon \tau \tilde{\eta}$

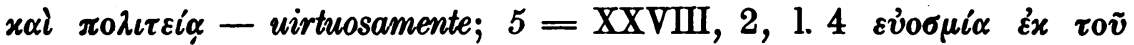

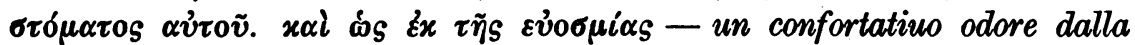

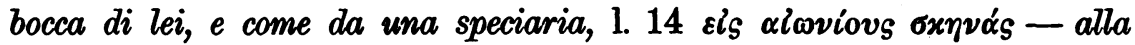

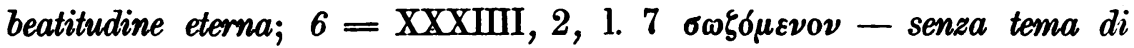

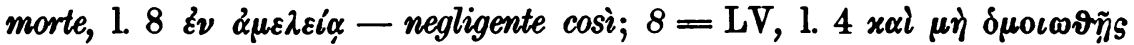

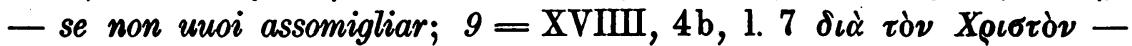
per amor di Christo; XVIII, 3d, 1. 3-4

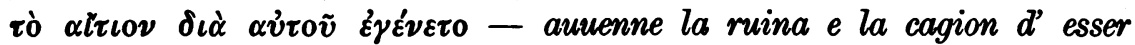

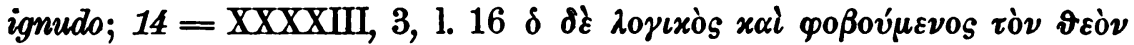

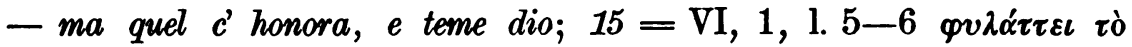

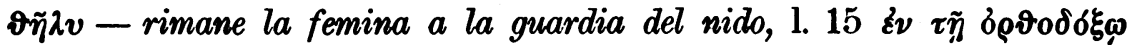

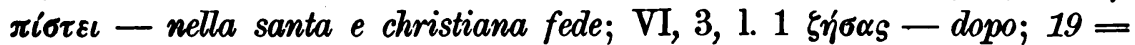

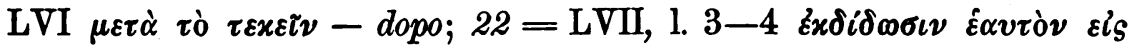

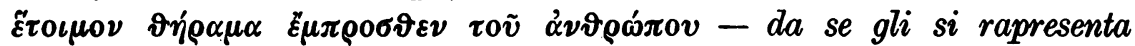

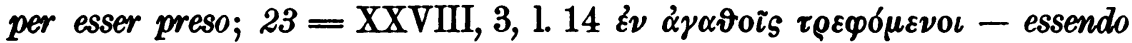

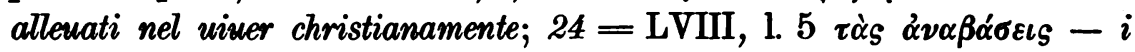

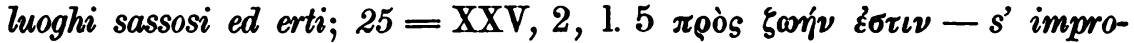

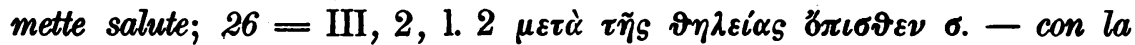

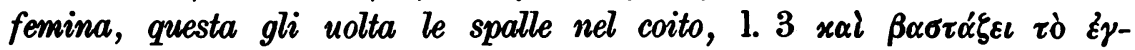

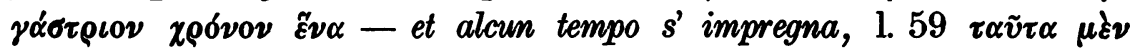

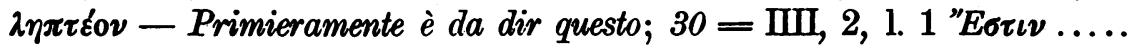

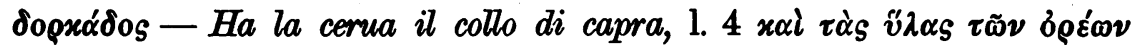

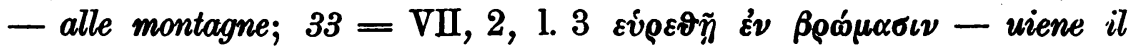
tempo di prender cibo.

Parole di $\boldsymbol{V p}$ rimangono in non pochi luoghi senza riscontro in $\boldsymbol{P}$ :

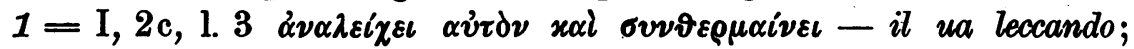

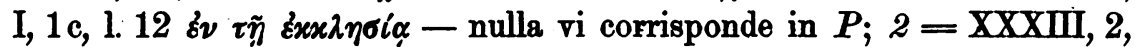
1. 1-2 manca in $P$ il principio sino a $\mu$ ov compreso: forse la cita-

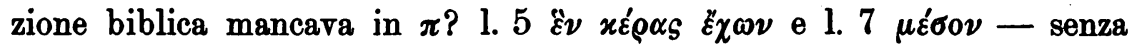
corrispondente traduzione in $P$. In pari condizione sono $3=\mathrm{LIII}$,

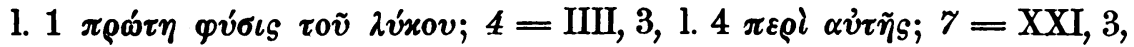

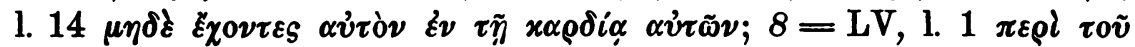

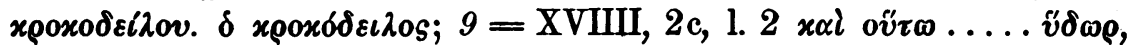

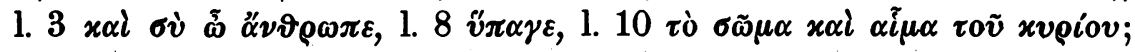

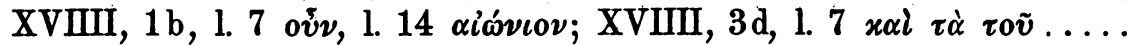

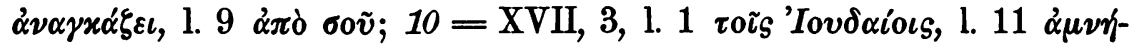

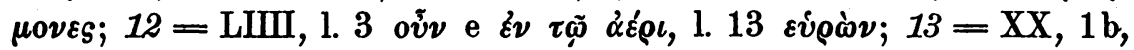

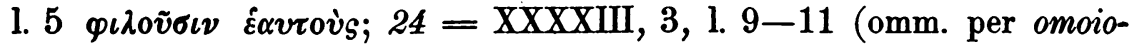

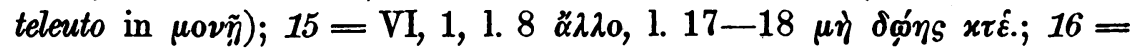

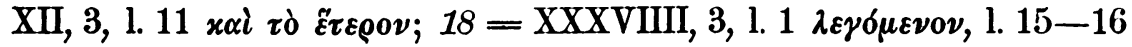


$\varepsilon \imath^{\prime} \tau \iota \varsigma$....

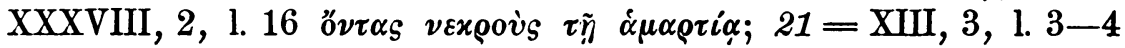

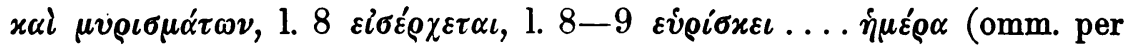

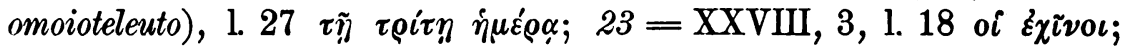

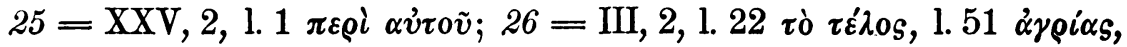

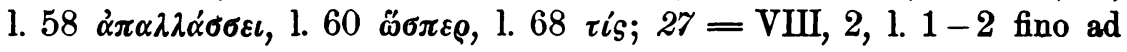

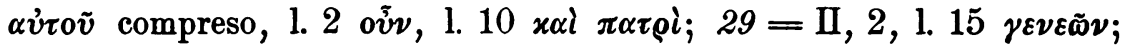

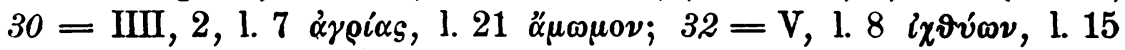

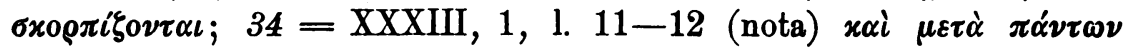

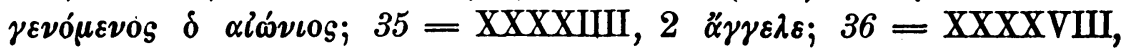

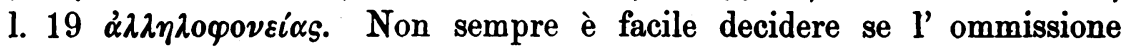
risalga solo a $P$ ovvero gia a $\pi$; ma qualche volta, oltre le citate, è palese che l' omoioteleuto fu la causa del fatto: $26=\mathrm{III}, 2,1.61-62$

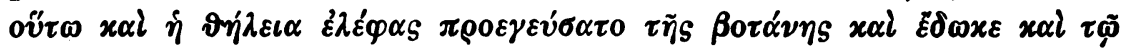

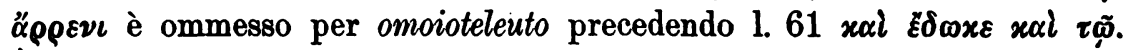
Eे ommessa in $\boldsymbol{P}$ sistematicamente la traduzione della parola ${ }^{~} E \rho \mu \eta \nu \varepsilon l \alpha$; il che potrebbe anche risalire a $\pi$ : infatti anche in $V$ la parola ${ }^{\circ} E \rho \mu \eta$ $\nu \varepsilon i \alpha$, scritta solitamente in rosso, manca in luoghi in cui l' ha $p ; \mathrm{p}$. es. in $3=$ LIII, 1.8 non c' è 'E $\mathrm{c}_{\mu \eta \nu \varepsilon l \alpha}$ neppure in $V$.

Speciale importanza hanno le differenze che si può supporre sieno derivate da differenza di lezione in $\pi$ o da differenza di lettura, trattandosi talora di non retta interpretazione de compendii grafici, specialmente alla fine delle parole, di spostamento d' accento, di punteggiatura

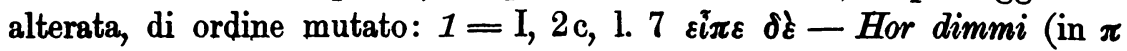

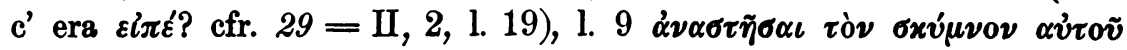
- risuscitare $i$ suoi figli da morte a vita (in $\pi$ c' era $\tau^{\backslash} \sigma x v \mu \nu \backslash$ ?),

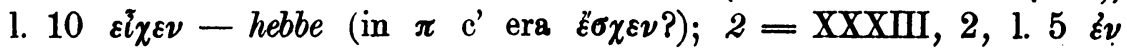

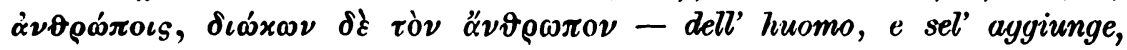

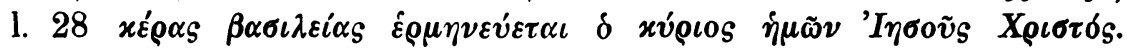
$\beta \lambda \dot{\varepsilon} \pi \omega \nu$ yà - il corno s' interpreta lo re. il Signor nostro Giesù Christo ueggendo (in $\pi$ c' era $\beta \alpha \sigma \iota \lambda \varepsilon i \alpha s$ ? e $\gamma \alpha \dot{\rho}$ era scritto in compendio?

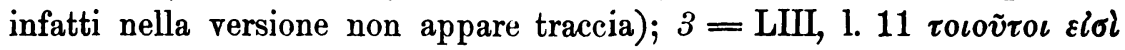

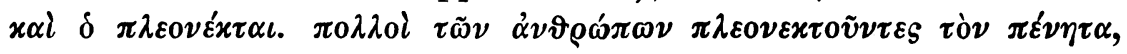
$x \alpha i$ of $\left.\pi \lambda \circ v_{\sigma \iota \iota} \dot{\alpha} \varrho \pi \alpha \dot{\xi}\right\} 0 v \sigma \iota$ - Tal ancho son molti huomini ingordi, $i$ quali ingannano il pouero et $i$ vicchi tolgono; $4=\mathrm{III}, 3,1.17^{\circ} \alpha \nu \delta \rho \alpha ́ \sigma \iota$

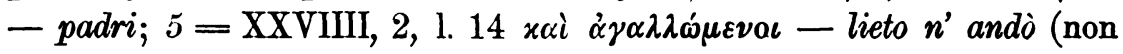

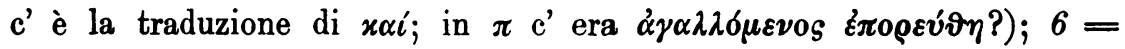

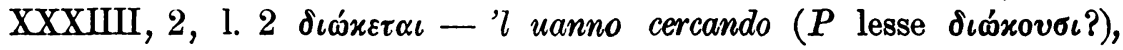

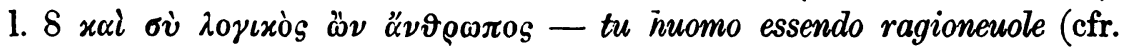
$15=\mathrm{VI}, 1,1.6 ; 18=\mathrm{XXXVIIII,} \mathrm{3,} \mathrm{1.} \mathrm{9;} 26=\mathrm{III}, 2,1.8), 1.14$ x

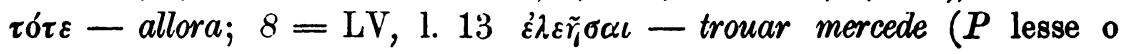




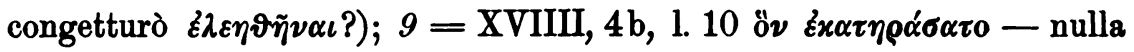
vi corrisponde nella versione del Pizzimenti; c' era scritto in $\pi \varepsilon^{2} x, \tau \eta$ -

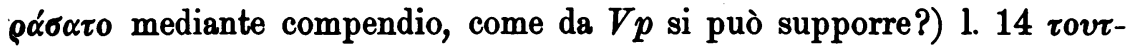

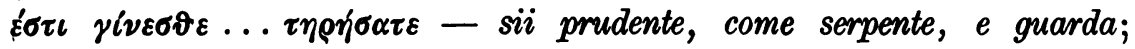

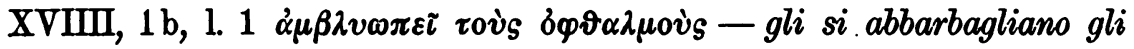

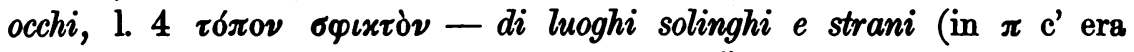
$\tau \delta \pi^{\prime} \sigma \varphi \iota x \tau \mid$ ?), $1.6 \beta \lambda \varepsilon \dot{\pi} \varepsilon \iota$ - considera ( $\beta \lambda \varepsilon^{\prime \prime} \pi$ in $\pi$ ?); $10=\mathrm{XVII}, 3$,

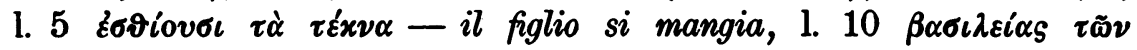

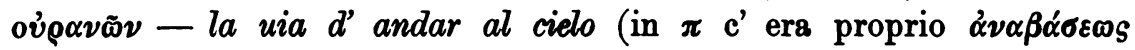

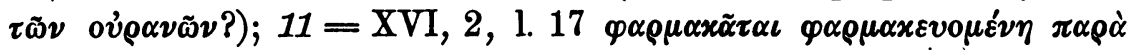

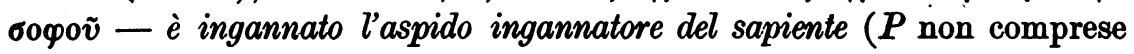

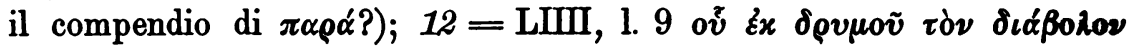

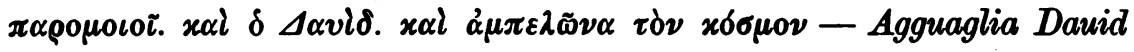

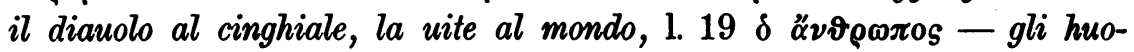
$\operatorname{mini}(\overline{\alpha \nu 0 S}$ o $\overline{\alpha \nu 0 \iota}$ in $\pi$ ?); $13=\mathrm{XX}, 1 \mathrm{~b}, 1.4 \pi 0 \rho \varepsilon v \varepsilon \tau \alpha \iota-u a n n o ;$

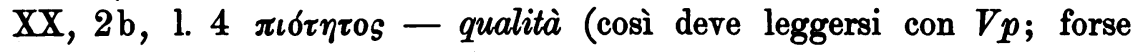

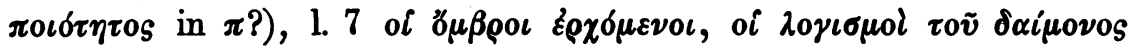
- $i$ pensieri del demonio, uenendo a guisa di pioggie ( $P$ lesse $\dot{\omega} S$

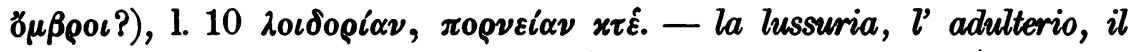
parlar molto, $l$ 'auaritia, $l$ 'ebbrezza (manca la traduzione di $\lambda o \iota \delta o \rho i \alpha \nu$, $\tilde{\varepsilon} \rho \iota \nu, \vartheta \vartheta \mu \delta \nu, x \varepsilon \nu \circ \delta \circ \xi \ell \alpha \nu$, ed inoltre $x \alpha \tau \alpha \lambda \alpha \alpha \lambda \iota \alpha \nu$ non è ben reso con $i\rceil$ parlar molto; in $\pi$ c' era $x \alpha \tau \alpha \lambda \alpha \lambda \alpha_{\alpha} \nu$ con il compendio per $x \alpha \tau \alpha$

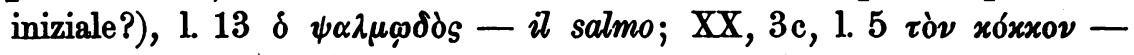
gli granelli; $14=\mathrm{XXXXIIII,} \mathrm{3,} \mathrm{1.} \mathrm{17-13} \mathrm{\pi 0 \lambda \lambda ol} \mathrm{.......} \mathrm{\lambda vं \psi o \nu \tau \alpha \iota} \mathrm{-}$ Et essendo in molte cose tentato, et hauendo patientia riceuerà maggior

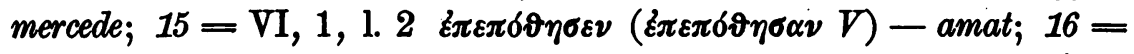

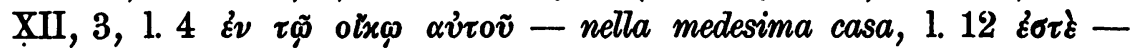
eritis; $17=\mathrm{XI}, 2 \mathrm{~b}, 1.1 \tau \tilde{\tau} \tau v_{\chi \eta}$ - naturalmente, $1.4 x \alpha \theta \eta \mu t \nu \eta-$ non

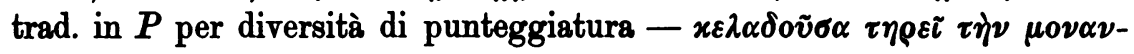

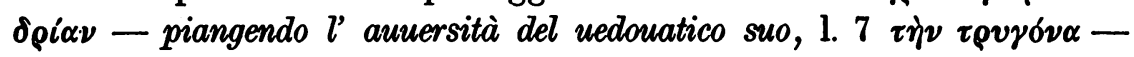

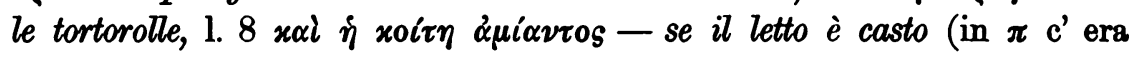

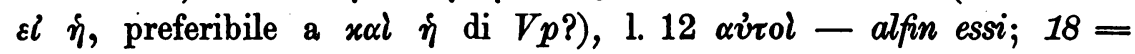

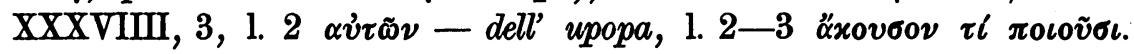

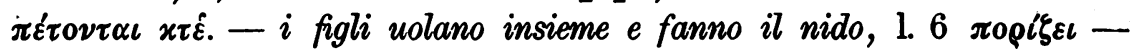

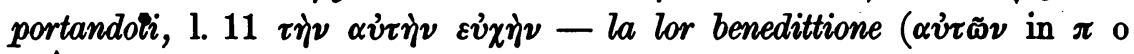

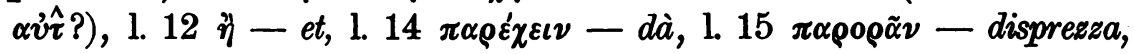
1. 28 iòv xúpıov - il figliuol (in $\pi \mathrm{c}^{\prime}$ era $\overline{x \nu}$ colla ben nota forma del $x$ simile al $\beta$ in forma quasi del nostro $u$ e del $\beta$ nel corsivo

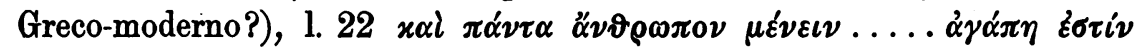
- e che ciascuno ami dio rettamente, credendo ch' egli $\grave{e} l$ ' amore,

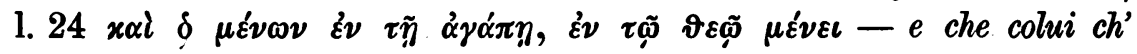




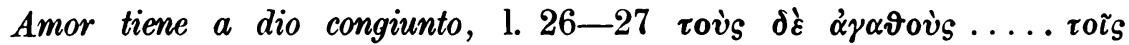
$\dot{\alpha} \gamma \alpha \vartheta 0 \tilde{I}_{S}-e t$ imiti $i$ buoni, e quei che seruono a dio giustamente e che

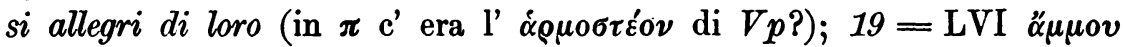

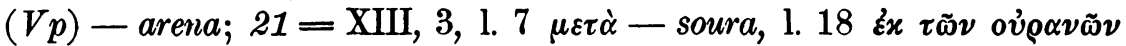

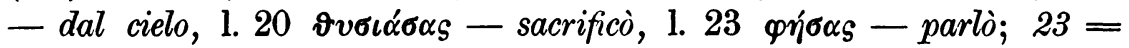

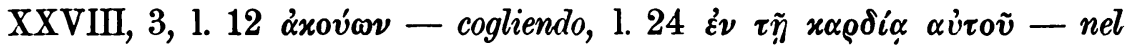

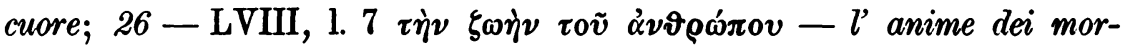

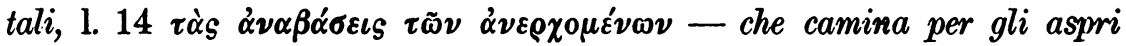

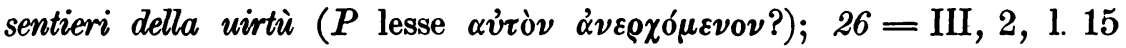

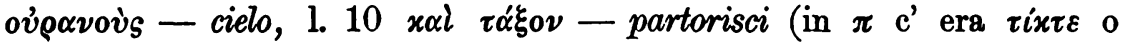

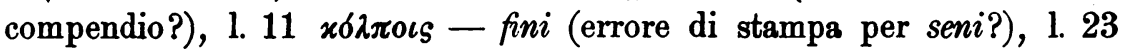

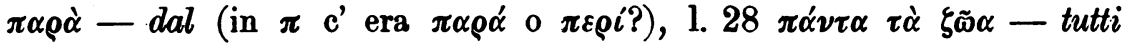

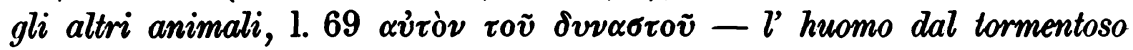

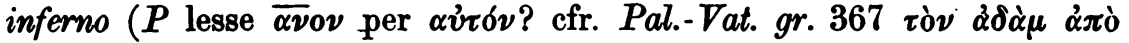

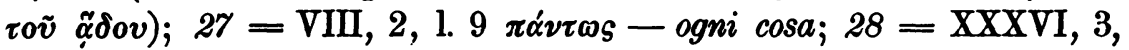

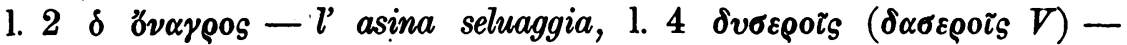

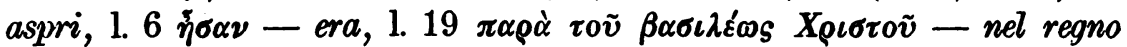
suo (in $\pi$ c' era compendio per $\pi \alpha \rho \alpha ́$ e $\left.\beta \alpha \sigma \iota \lambda \varepsilon \varepsilon^{\prime}\right)_{s}$ ?); $29=\mathrm{II}, 2,1.23$ $\dot{\eta} \dot{\eta} \delta \circ \nu \eta-i$ piaceri; $30=\mathrm{IIII}, 2$, l. $2 \xi \dot{\eta} \sigma \alpha \sigma \alpha-u i u e$ (nel Pal.-Vat.

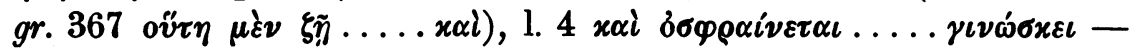
et odorando il pertugio dou' $\grave{e}$ ascoso $l$ 'angue, annusando $i l$ conosce,

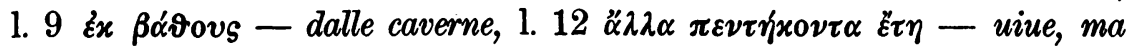

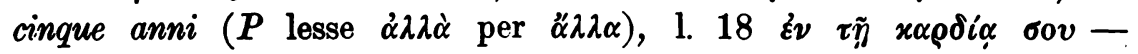
posposto in $P, 1.19 \varphi \lambda \varepsilon^{\prime} \beta \alpha s$ - chiaro lume, $1.21 \tau \tilde{\eta} \mu \varepsilon \tau \alpha \nu o i \alpha$ - in

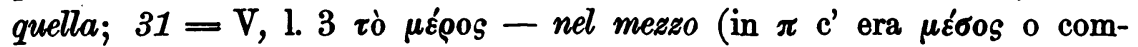

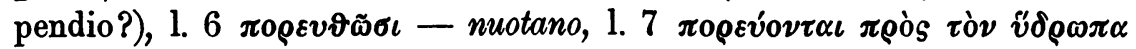
- uanno appresso $l$ ' hydrope (non letto il compendio di $\pi \varrho o ́ s ?) ; 32=$

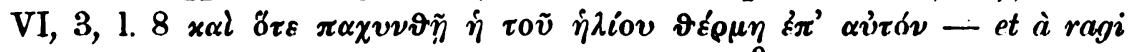
di lui riscaldandosi (a $P$ recava difficoltà $\pi \alpha \chi v \boldsymbol{v}$ ?); $33=$ VII, $2,1.11$ $\delta \iota \alpha \mu \varepsilon v_{v} \iota$ - sarà serbato (in $\pi$ c' era $\delta \iota \alpha \mu \varepsilon v \varepsilon \tilde{\imath} ?$ ); $34=\mathrm{XXXIII,} \mathrm{1,} \mathrm{1.} 3$

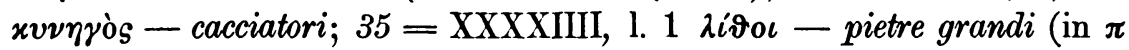

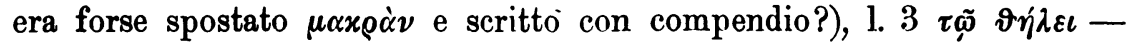

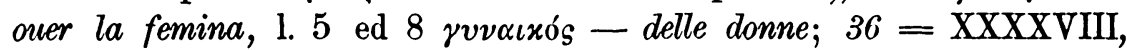

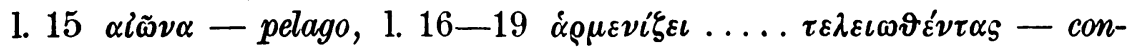
tende con $i$ legni di giungere innanzi tempo a riua, ma non perseuera insino all estremo: $e$ se comincia le buone opere, $\overline{10}$ le reca a termine, 1. $20 x v ́ \mu \alpha \tau \alpha-p e r ~ l ' o n d e$ (in $\pi$ c' era $x v \mu \alpha^{\tau}$ ? la parola in $P$ è collegata ad $\tilde{\varepsilon} \nu \varepsilon x \alpha$ ed il passo è frainteso); $37=\mathrm{XXVI}, 1.4$ v $\pi \dot{\varepsilon} \varrho \tau \tilde{\omega} \nu$

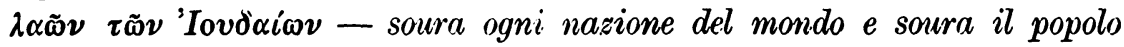
de' Giudei $(\boldsymbol{P}$ è d' accordo con $v$; vero è che il $\boldsymbol{P}$ poteva rammentarsi consimili locuzioni bibliche), 1. 1C-13 mancano in $P$. 
Sono importanti quei luoghi nei quali $P$ si accorda solo con $p$ o

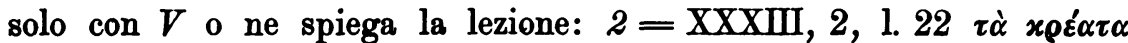

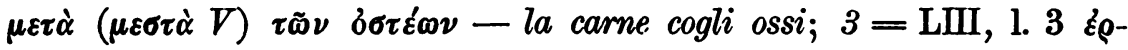

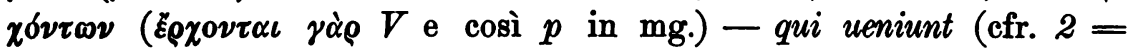

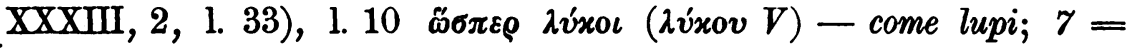
XXI, 3, 1. 6-8 la ommissione di $V$, per omoioteleuto, è confermata

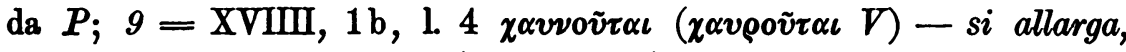
1. 9 v il primo mandato del paradiso: $e$ se $l$ ' hauesse osseruato, non sarebbe

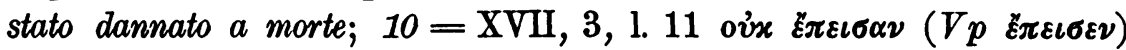
av่zò̀s - ai quali essi non hauendo creduto; $11=\mathrm{XVI}, 2,1.6$ ஸs

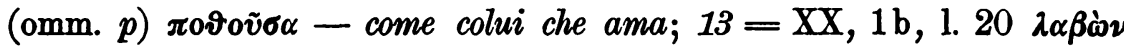

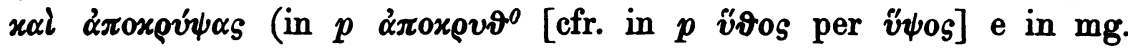
$x \rho v ́ \psi \alpha s)-c$ ' hauendo riceuuto il talento, $l$ ' hai nascoso sotterra ( $\pi$ era

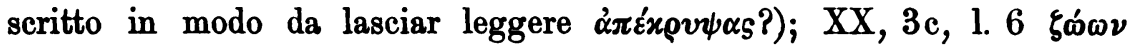

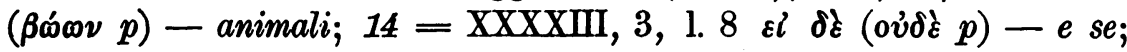

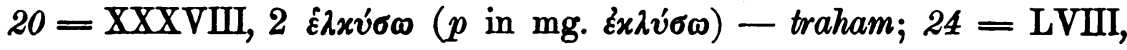

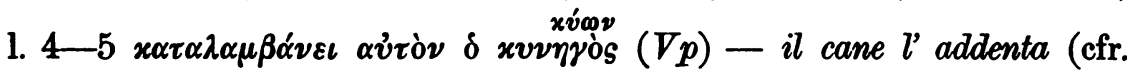

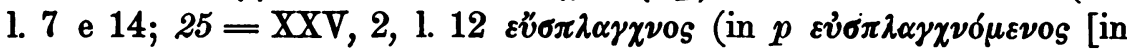
mg. $\varepsilon \tilde{v} \sigma \pi \lambda \alpha \gamma \chi \nu 0 s]$ nato da errata interpretazione del compendio) -

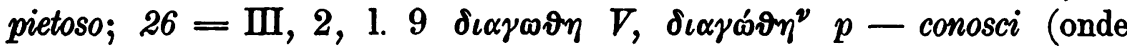

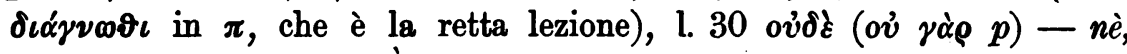

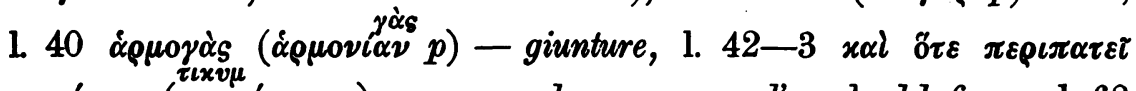
$x v \mu \alpha \tau^{\prime} \omega \nu(\tau \mu v \tau \alpha \dot{\tau} \omega \nu p)-e$ quando scorre per $l$ ' onde del fume, 1.68

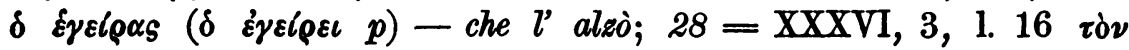

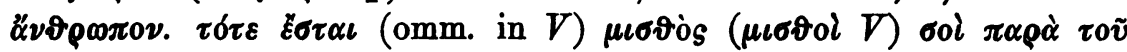
$K v p l o v-l$ huomo alle uolte dal signor approuato (in $\pi$ non $c^{\prime}$ era E $\sigma \tau \iota$, che manca anche in $V$, e $\mu \iota \sigma \vartheta \grave{s}$ бol fu letto $\mu \iota \sigma \vartheta \omega \tau \delta$ s?); $30=$ IIII, 2, l. 7 (nota) cls oíov Bávos - e se l' serpente giace sotterra (letto $\varepsilon l$ per $\varepsilon l_{S}$ ); $31=\mathrm{V}, 1.2 x \dot{\eta} \tilde{r o v}$ (così oltre $V p$ anche il Vat.-Pal. gr.

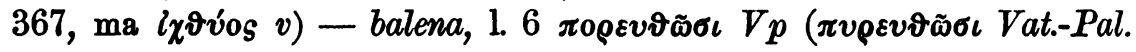

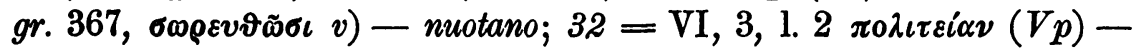

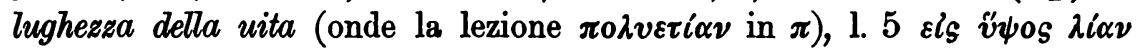

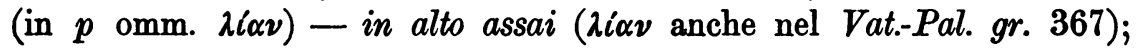

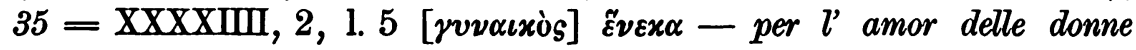

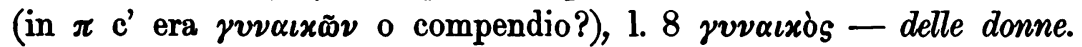

Ma di gran lunga i più notevoli sono quei passi di $P$ che completano lacune od ommissioni di $V p: 23=\mathrm{XXVIII,} \mathrm{3,1.18 \delta}$

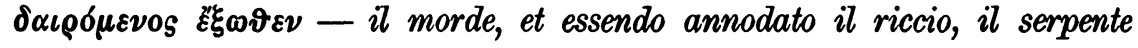
di fuori trafitto ( $P$ ha più di $V p$ dove si può supporre un' ommissione 
derivante da omoioteleuto in $\delta$ Ex $\chi$ ĩvos); $28=\mathrm{XXXVI}, 3,1.12-13$ in

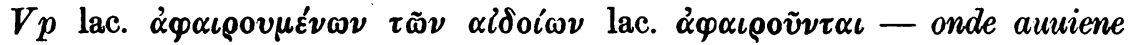
che molti priui delle parti uergognose, anchora son della uita priuati (in

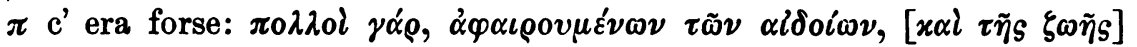
$\dot{\alpha} \varphi \alpha \iota \rho o \tilde{v} \nu \tau \alpha \iota)$. Le linee $1-9$ del capo $29=\mathrm{II}, 2$ si possono completare così:

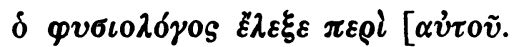

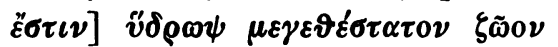

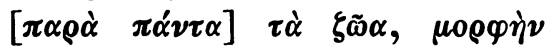

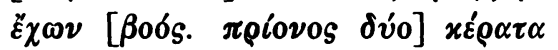

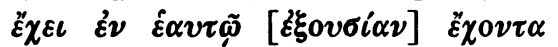

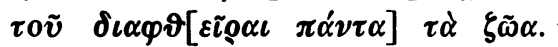
$x \alpha i$ ov่dtे $\pi \rho[0 \sigma \varepsilon \gamma \gamma i \xi o v \sigma \iota \nu \alpha \dot{v} \tau \tilde{\omega}]$ $\tau \grave{\alpha} \xi \tilde{\omega} \alpha . \dot{\alpha} \lambda \lambda \dot{\alpha} \mu \varepsilon \gamma[\varepsilon \vartheta \hat{\varepsilon} \sigma \tau \alpha \tau \alpha \delta \varepsilon \dot{\nu} \nu \delta \alpha$

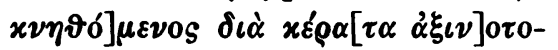

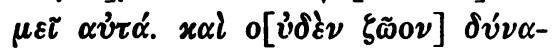

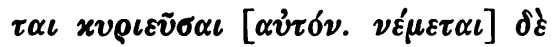

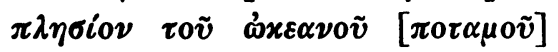

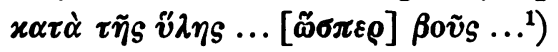

Il Physiologo dice dell' hydrope. $E l$ hydrope animal di grandezza soura tutti gli altri animali. haue il uolto di toro, ed in testa due corna simili a quelli del prione potenti ad uccidere tutte le fiere: le quali per la paura non gli si appressano miga. e per la grandezza strebiando gli alberi con le corna li sega e gli taglia. $E$ nissum animal può signoreggiarlo. Pasce $l$ ' hydrope uicino al fiume Oceano ne' boschi ... come bue ...

A questi passi si può arvicinare una serie di citazioni bibliche che trouandosi in $P$, e potendo risalire a $\pi$, o non si trovano affatto in $V p$, o vi si trovano più abbreviate: vero è che spesso $P$ dà le citazioni bibliche in latino, e che per le citazioni bibliche sia nella versione, sia nel testo di essa la memoria poteva avere la sua parte, non però così grande come parrebbe a prima vista: ciò invero risulta studiando le citazioni bibliche nelle varie redazioni, non solo greche, del Physiologus. Cfr. $3=\mathrm{LIII}, 1.4$ con $\alpha{ }^{\rho} \pi \alpha \gamma \varepsilon S$ in $V p$ finisse la citazione biblica, ma $\boldsymbol{P}$ prosegue: $E t$ ecce mitto uos tanquam oues in medio lupo$\mathrm{rum} ; 6=\mathrm{XXXIIII,} \mathrm{2,} \mathrm{1.} \mathrm{9-10} \mathrm{घ̈xxо \psi о \nu} \alpha \dot{v} \tau \dot{\eta} \nu$ - tagliala tosto; $7=$

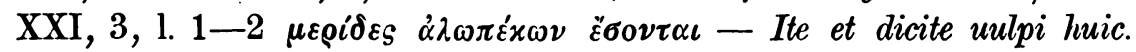
ecce eijcio demonia. e Dauid ne' salmi dicendo; $9=\mathrm{XVIIII}, 4 \mathrm{~b}, 1.1-2$

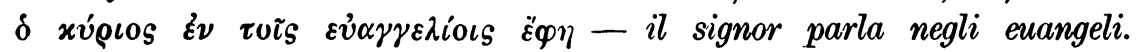

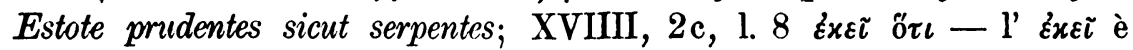
omm. nella uersione, che per questo passo è in italiano; $10=\mathrm{XVII}, 3$

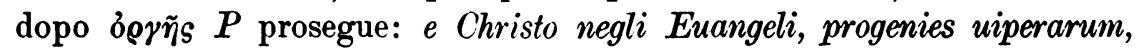
quomodo potestis bona loqui, cum sitis mali?; $11=\mathrm{XVI}, 6,1.1 \pi \varepsilon \rho 1$

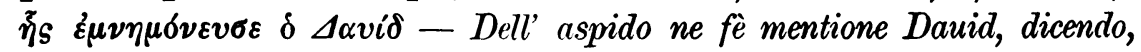
sicut aspides surdi, et obscurantis aures suas; quae non exaudiet uocem

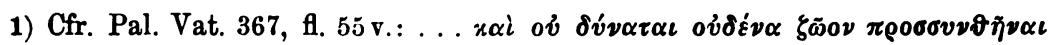

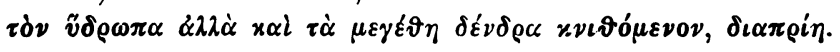


incantantium et uenefici incantantis sapienter; $12=\mathrm{LIIII}, 1.1-2 \pi \varepsilon \rho i$ $\check{\omega} \nu \ldots . . \tau \dot{\eta} \nu \dot{\alpha} \mu \pi \varepsilon \lambda \tilde{\omega} \nu \alpha \dot{\alpha} \mu o v-D a u i d$ fè mentione del cinghiale, dicēdo. Exterminauit eam aper de sylua; et singularis forus depastus est eam;

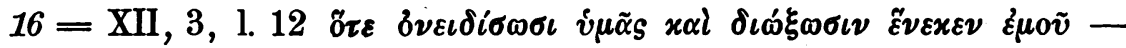
cum uos oderint homines, et cum separauerint uos, et reprobauerint, et eiecerint nomen uestrum tanquam malum, propter filium hominis; $20=$ XXXVIII, 2, 1.1 P incomincia: dell' erodio fè mentione Dauid ne' salmi; dicendo: herodij domus dux est eorum. montes excelsi ceruis, petra refugium herinacei. $E l$ erodio caro ài figli suoi ecc. ( $\pi$ incominciava:

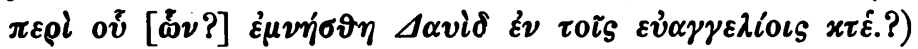

Tralascio altre differenze, che pur è bene non vengano dimenticate, e passo ad un' altra serie di considèrazioni.

La traduzione del Pizzimenti non sempre corrisponde al testo che noi abbiamo e che si può supporre esistesse anche in $\pi$; la causa talora può essere attribuita a confusione e ad errore, non di sola lettura: $7=\mathrm{XXI}, 3 \pi \varepsilon \iota \nu \alpha \dot{\sigma} \sigma \iota$ - ha beuuto, 1. $13 \tau i \delta \rho \alpha^{\prime} \xi 0 \nu \tau \alpha \iota \tau o \tilde{v} \pi \lambda \eta-$

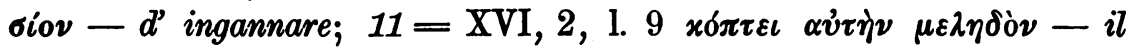

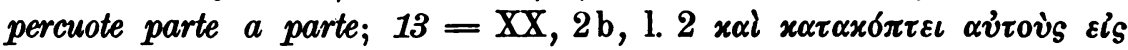
$\mu \varepsilon ́ \sigma o \nu$ - e gli aduna insieme; $19=\mathrm{LVI}, 1.7$ d $\mu \nu \eta \dot{\mu} \mu \nu \varepsilon s$ - disamore-

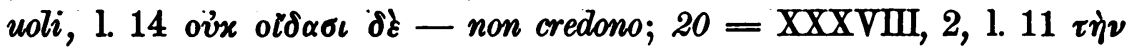

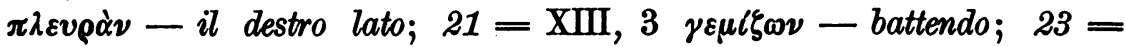

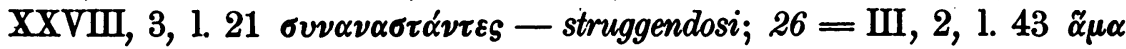

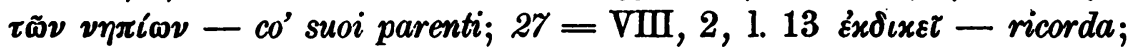

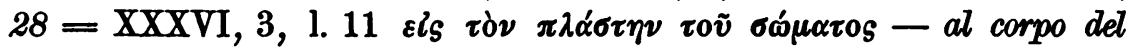
fattor nostro (in $\pi$ c' era $\pi \lambda \alpha \sigma \hat{\tau}$ e $\sigma \omega \mu \mu^{i} ?$ ?), $1.17 \pi \alpha v_{\varepsilon \iota} \pi 0 \lambda \varepsilon \mu 0 \tilde{\sigma} \sigma \alpha-$

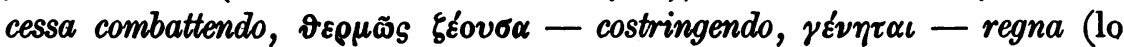

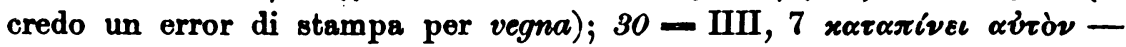
ci suga il sangue; $36=\mathrm{XXXXVIII,} 1.4 \hat{\eta} \lambda^{\prime} \grave{\eta} \mu^{\prime}-$ ondeci $\partial$ dodeci.

$\mathrm{Da}$ ultimo richiamo l' attenzione sui luoghi seguenti: $8=\mathrm{LV}$, 1. 3-4 $\delta \varphi \vartheta \alpha \lambda \mu o \tilde{v}-u e l l i c o$ (deve realmente leggersi $\delta \mu \varphi \alpha \lambda o \tilde{v}$ ); $11=$

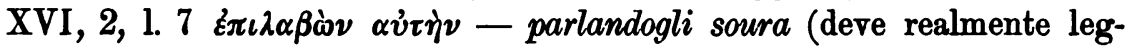
gersi $\dot{\varepsilon} \pi \iota \lambda \alpha \lambda \tilde{\omega} \nu) ; 30=\mathrm{III}, 2,1.9 \dot{\varepsilon} \lambda \varepsilon \tilde{\nu}-$ il passo etimologico in $P$ è scritto in lettere greche, ma colla grafia $\ddot{\varepsilon} \lambda \varepsilon \iota \nu$; è però molto istruttivo che il passo greco sia trascritto perchè ci dà $\varepsilon^{\prime} x \beta \alpha^{\prime} \vartheta 0 v s$, mentre la versione traduce - dalle cauerne: se il passo greco non fosse trascritto, arremmo dovuto domandarci se il $\boldsymbol{P}$ avesse, erroneamente o meno, per la presenza di compendio finale o per altra causa, letto $\beta \alpha \vartheta \tilde{\omega} \nu$ invece di

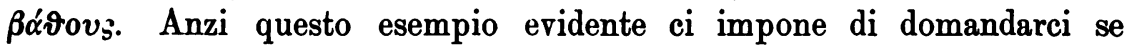
consimili differenze fra la versione ed il testo abbiano sempre la medesima causa: si dovrà concludere che alle differenze contribuì anche 
l' intenzione del traduttore, nel quale si deve ammettere una certa libertà ed anche la capacità di correzioni e di congetture di fronte al testo. Però il numero dei casi dianzi veduti e la natura loro ci autorizzano a dire che molte volte la differenza è dovuta alle condizioni in cui si trovava $\pi$, del quale ora possiamo avere un' idea abbastanza chiara.

È troppo evidente che $\pi$, il ms. greco usato dal Pizzimenti, non era nè $V$ nè $p$, nè una copia di $V$ o di $p$; più che dal minor numero di capitoli contenuti in $\pi$ la cosa è provata dal fatto che con $\pi$ si completano le lacune di $V$ e $p$ : il che dimostra che $\pi$ era in migliori condizioni del ms. onde derivarono $V$ e $p$. E questa circostanza ci induce a credere che $\mathrm{i}$ capitoli che $V$ e $p$ hanno in piu di $x$, non si trovassero in $\pi$, e non vi si trovassero pur essendo $\pi$ completo e completa la versione del Pizzimenti. A meglio spiegarmi, il Pizzimenti diede nella sua versione tutto quello che trovò in $\pi$, e $\pi$ presentava un minor numero di capitoli di fronte a $V$ e $p$, perchè i capitoli mancanti in $\boldsymbol{P}$ (ed in $\boldsymbol{\pi}$ ) sono privi dei caratteri della redazione Basiliana, per quanto in $\pi$ si trovasse il capitolo 32 (da unirsi al 15 di $P$ per rifare il 15 di $V p$ ) privo anch' esso dei caratteri della redazione Basiliana. Il che ci formisce la prova che il Pizzimenti non fece egli una scelta attenendosi al corpo Basiliano e lasciando il resto. C' è anzi una ragione per credere che la terza natura dell' aquila, qual' è data da $V$ e $p$, dopo la prima e la seconda natura, e da $\boldsymbol{P}$ a distanza di molti capitoli, abbia il suo posto originario in $P(\mathrm{e} \pi)$ e non in $V$ e $p$, dacchè la redazione Basiliana dichiara espressamente di occuparsi di sole due nature dell' aquila (Stud. Ital. di Fil. class. V, p. $136-$

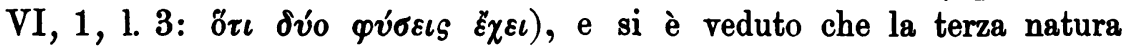
$(-32$ di $P$ ) rassomiglia ai capitoli che in $p$ presentano tutbora le traccie anche esterne di un' origine differente, rassomiglia cioè ai capitoli 26 (terza natura) - 48 di $V$ e $p$, dai quali è però da escludere il 32 di $p$, che è della redazione Basiliana. In condizioni opposte a quelle del capitolo 31 di $P$ si trova il capitolo 37 , parte seconda, di $P$, cioè del pelicano: le due nature si trovano in $V$ e $p$ separate ai capitoli 27 e 41, e il 27 è della redazione Basiliana e il 41 è di altra redazione. Vale a dire per l' Aquila $V$ e $p$ presentano uniti tutti gli elementi eterogenei, e $P$ li dà separati; invece per il Pellicano gli elementi eterogenei sono separati in $V$ e $p$ e riuniti in $P$. Nè può dirsi questa una riunione o separazione intenzionale di $P$ o di $V$ e $p$; ma è piutiosto da credersi o che al corpo originario della redazione Basiliana si andarono facendo, in differenti manoscritti, aggiunte successive dedotte da altre redazioni (e così si spiegherebbero i non pochi dupli- 
cati), o che l' autore della redazione Basiliana non fini la sua elaborazione, ma, presa a fondamento una redazione del Physiologus ricchissima di capitoli, a parecchi sorrappose la veste Basiliana, ad altri no, e quest' ultimi, dapprima aggregati al corpo Basiliano, ne furono man mano eliminati. Pur riservando qualunque giudizio sulla derivazione della redazione Basiliana da questa o quell' altra redazione del Physiologus, e sul collegamento di essa a questa o quell' altra redazione, la prima ipotesi appare di gran lunga la piu verisimile ed ha per sè, sin d' ora, parecchi argomenti, che uno studio ulteriore metterà maggiormente in luce e conforterà di altre prove. ${ }^{1}$ )

Tornando a $\pi$, s' è veduto che esso differisce da $V$ e $p$ e rassomiglia a $V$ e $p$ nella misura e nella maniera in cui $V$ e $p$ presentano rassomiglianze e differenze fra di loro; anzi i fatti più notevoli a questo proposito si possono ricondurre nella più parte dei casi a fenomeni grafici, specialmente a compendii e abbreviazioni, talchè $\pi, V, p$ appaiono derivati da un medesimo manoscritto fondamentale, di non facile lettura e ricco di compendii grafici. $E$ ciò spiega le incertezze, le somiglianze, le dissomiglianze dei tre mss. Ma se per $V$ e $p$ è possibile ammettere la derivazione da uno stesso e identico manoscritto, $\pi$ invece ha di fronte al comune archetipo $\Phi$ una derivazione più vicina che $V$ e $p$. Indicando con $\Pi$ il ms. onde $\pi$ fu trascritto, e con $\beta$ il manoscritto di cui $V$ e $p$ sono copia, arremmo il seguente stemma

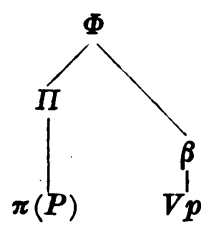

La linea a sinistra, da $\Phi$ a $\Pi$, più corta che la linea a destra, $\operatorname{tra} \Phi$ e $\beta$, dimostra che le infiltrazioni furono minori in $\Pi$ che in $\beta$, minori quindi in $\pi, P$ che in $V$ e $p$, per quanto $\Pi$ e $\beta$ fossero coevi e, a mio credere, fra il secolo $\mathrm{X}$ ed il $\mathrm{XII}$.

Torino.

C. 0. Zuretti.

1) Cfr. $M$ Goldstaub, in B. Z. VIII, p. $529 \mathrm{seg}$. 